\title{
Endogenous small-noncoding RNAs and their roles in chilling response and stress acclimation in Cassava
}

Jing Xia ${ }^{1,3+}$, Changying Zeng ${ }^{2 \dagger}$, Zheng Chen ${ }^{1,3}$, Kevin Zhang ${ }^{1,3}$, Xin Chen ${ }^{2}$, Yufei Zhou ${ }^{2}$, Shun Song ${ }^{2}$, Cheng Lu², Ruiju Yang ${ }^{2}$, Zi Yang ${ }^{2}$, Junfei Zhou' ${ }^{1}$, Hai Peng ${ }^{1}$, Wenquan Wang ${ }^{2}$, Ming Peng ${ }^{2^{*}}$ and Weixiong Zhang ${ }^{1,3,4^{*+}}$

\begin{abstract}
Background: Small noncoding RNA (sncRNA), including microRNAs (miRNAs) and endogenous small-interfering RNAs (endo-siRNAs) are key gene regulators in eukaryotes, playing critical roles in plant development and stress tolerance. Trans-acting siRNAs (ta-siRNAs), which are secondary siRNAs triggered by miRNAs, and siRNAs from natural antisense transcripts (nat-siRNAs) are two well-studied classes of endo-siRNAs.

Results: In order to understand sncRNAs' roles in plant chilling response and stress acclimation, we performed a comprehensive study of miRNAs and endo-siRNAs in Cassava (Manihot esculenta), a major source of food for the world populations in tropical regions. Combining Next-Generation sequencing and computational and experimental analyses, we profiled and characterized sncRNA species and mRNA genes from the plants that experienced severe and moderate chilling stresses, that underwent further severe chilling stress after chilling acclimation at moderate stress, and that grew under the normal condition. We also included castor bean (Ricinus communis) in our study to understand conservation of sncRNAs. In addition to known miRNAs, we identified 32 (22 and 10) novel miRNAs as well as 47 (26 and 21) putative secondary siRNA-yielding and 8 (7 and 1) nat-siRNA-yielding candidate loci in Cassava and castor bean, respectively. Among the expressed sncRNAs, 114 miRNAs, 12 ta-siRNAs and 2 nat-siRNAs showed significant expression changes under chilling stresses.

Conclusion: Systematic and computational analysis of microRNAome and experimental validation collectively showed that miRNAs, ta-siRNAs, and possibly nat-siRNAs play important roles in chilling response and chilling acclimation in Cassava by regulating stress-related pathways, e.g. Auxin signal transduction. The conservation of these sncRNA might shed lights on the role of sncRNA-mediated pathways affected by chilling stress and stress acclimation in Euphorbiaceous plants.
\end{abstract}

Keywords: microRNA, tasiRNA, chilling acclimation, Cassava

\section{Background}

Multicellular eukaryotes develop diverse small noncoding RNA (sncRNA) mechanisms for gene regulation at both DNA and RNA levels. In plants, most sncRNAs are generated by RNase III-type endonuclease DICERs or DICER-LIKE (DCL) proteins, and then incorporated into

\footnotetext{
* Correspondence: mmpeng_2000@yahoo.com; weixiong.zhang@wustl.edu ${ }^{\dagger}$ Equal contributors

${ }^{2}$ The Institute of Tropical Bioscience and Biotechnology, Chinese Academy of Tropical Agricultural Sciences, Haikou, China

${ }^{1}$ Institute for Systems Biology, Jianghan University, Wuhan, Hubei 430056, China

Full list of author information is available at the end of the article
}

ARGONAUTE (AGO) proteins to exert their gene regulatory functions at the transcriptional level through DNA methylation and/or histone modification, and at the posttranscriptional level by mRNA cleavage, mRNA degradation or translational repression [1-5].

MicroRNAs (miRNAs) and endogenous small-interfering RNAs (endo-siRNAs) are two major classes of sncRNAs. miRNAs are typically processed from RNA polymerase II transcripts that fold into hairpin structures [6]. In plants, such hairpin-structured pre-miRNAs are then processed by DCL proteins in the nucleus to release $\sim 22$-nt doublestranded RNAs with $\sim 2$-nt 3' overhangs, namely miRNA/

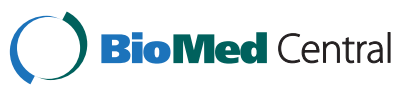

(c) 2014 Xia et al.; licensee BioMed Central Ltd. This is an Open Access article distributed under the terms of the Creative Commons Attribution License (http://creativecommons.org/licenses/by/4.0), which permits unrestricted use, distribution, and reproduction in any medium, provided the original work is properly credited. The Creative Commons Public Domain Dedication waiver (http://creativecommons.org/publicdomain/zero/1.0/) applies to the data made available in this article, unless otherwise stated. 
miRNA* duplexes. The mature miRNAs are then loaded into the AGO-containing RNA-induced silencing complexes (RISC) in the cytoplasm to exert their regulatory effect by guiding the RISC to target transcripts through complete or partial complementary base pairing [7].

Endo-siRNAs have more diverse sources of origin. Endo-siRNAs arise from long double stranded RNAs (dsRNAs), which are formed from overlapped antisense transcripts or products of RNA-dependent RNA polymerases (RdRP) [8-11]. In plants, different classes of endo-siRNAs have been described based on their distinct characteristics, biogenesis pathways and functions $[3,12]$. Trans-acting siRNAs (ta-siRNAs) and siRNAs from natural antisense transcripts (nat-siNATs) are two major classes of endo-siRNAs. Typically 21-nt in length and arranged in registers of 21-nt long phasing [7], tasiRNAs are generated by phased Dicer processing of noncoding TAS genes or mRNA transcripts initiated by miRNAs [13-16].

Several genic regions have been annotated as ta-siRNAyielding loci. In Arabidopsis, for example, a TAS3 gene has a pair of miR390 binding sites, which define and produce a single RNA strand that is subsequently turned into a double stranded RNA by RDR6 polymerase. The double stranded RNA is subsequently cleaved by DCL4 to release a series of $\sim 21$-nt phased ta-siRNAs. Among these tasiRNAs, one ta-siRNA species, named tasiARF, is broadly conserved and targets genes in the ARF family in trans [13]. Moreover, Arabidopsis has three TAS3 homologues $(A t T A S 3 a / b / c)$ [16], rice carries three TAS3 loci [17], and $P$. patens has four $(P p T A S 3 a / b / c / d)$ [13]. Besides TAS3, three other TAS genes (TAS1, TAS2 and TAS4) have been reported in Arabidopsis, while TAS3 is the only one broadly conserved, from rice and Arabidopsis to P. patens and Cassava [13]. ta-siRNAs may also arise from coding genes; a large number of genes encoding nucleotide binding site-leucine-rich repeat (NBS-LRR) plant innate immune receptors have been reported to give rise to ta-siRNAs, which were triggered by miR482 and $\operatorname{miR} 2118[16,18,19]$.

Endo-siRNAs can also be generated from cis-natural antisense transcripts (cis-NATs) [20-26]. These siRNAs, named as nat-siRNAs, can be induced by abiotic and biotic stresses $[20,27,28]$ or can accumulate in specific developmental stages [23,24]. The biogenesis of salt- and bacterium-induced nat-siRNAs in Arabidopsis requires DCL1 and/or DCL2, RDR6, and Pol IV [20,28]. For example, the expression of ARIADNE14 is de-repressed in dcl1, hen1, hyl1, sde4, rdr2 and sgs3 mutants, suggesting that the nat-siRNAs from the cis-NAT pair ARIADNE14 and KOKOPELLI are dependent of DCL1, HEN1, HYL1, RDR2, SGS3 and PolIV [23].

Despite the broad existence of ta-siRNAs and natsiRNAs in plant species, many of their features remain to be studied. It requires further effort to gain a comprehensive view of the genomic loci where ta-siRNAs and nat-siRNAs arise and to understand their regulatory roles in adaptation to dynamic environmental conditions. Further, little is known about the expression of miRNAs and endo-siRNAs in Euphorbiaceous plants. Here, we performed a comprehensive study of miRNAs, ta-siRNA and nat-siRNA in two agri-economic important Euphorbiaceous plants, Cassava (Manihot esculenta) and castor bean (Ricinus communis). Euphorbiaceae is one of the largest families in Angiosperms, consisting of more than 300 genera and about 7,500 species. Cassava is the most important crop in Africa and Southeast Asia and a primary source of food in most parts of the regions. Castor oil from castor bean has a medical use and is a critical raw material for many industrial products, such as lubricants and paints. Cassava and castor bean are capable of high photosynthesis and have complex traits for adapting to dynamic environments, making them ideal for studying small-RNA mediated gene regulation in stress response and stress acclimation. In the current study, we focus on identification and characterization of miRNAs, ta-siRNAs and nat-siRNAs in these two plants, particularly in Cassava, in response to chilling stress and in the process of chilling acclimation.

\section{Results}

\section{Experiments to explore chilling response and stress acclimation}

The study of miRNAs and endo-siRNAs in Cassava and castor bean was based on an integrative sncRNA and mRNA expression profiling experiment (Additional file 1: Figure S1 and Methods) [29]. Briefly, SC124, which is sensitive to chilling and is a widely planted Cassava cultivar in China, was subjected to three chilling stress treatments. The first was gradual chilling acclimation (CA) at a moderate stress where plants were subjected to a temperature decrease from $24^{\circ} \mathrm{C}$ to $14^{\circ} \mathrm{C}$ by $-2^{\circ} \mathrm{C} / \mathrm{h}$ and then grew for five days. In the second treatment of chilling stress after chilling acclimation (CCA), plants after the CA treatment were transferred further from $14^{\circ} \mathrm{C}$ to $4^{\circ} \mathrm{C}$ by $-2^{\circ} \mathrm{C} / \mathrm{h}$ and cultivated for another 5 days. In the third experiment, plants were subjected to chilling shock (CS) by a dramatic temperature drop from $24^{\circ} \mathrm{C}$ to $4^{\circ} \mathrm{C}$ with a gradient of $-4^{\circ} \mathrm{C} / \mathrm{h}$. For comparison, plants grown under $24^{\circ} \mathrm{C}$ were taken as the normal control (NC). It is important to note that the three chilling treatments resulted in distinct phenotypes of elevated leaf proline content and/or malondialdehyde content [29].

\section{sncRNA and mRNA expression profiling by Next Generation sequencing}

Four small-RNA libraries from the chilling-treated (i.e., $\mathrm{CA}, \mathrm{CCA}$ and $\mathrm{CS}$ ) and the normal (i.e., NC) plants of 
SC124 were prepared and sequenced separately using Illumina Genome Analyzer IIx (GAIIx) (see Methods, sequencing data in NCBI/GEO, accession \# GSE52178). These libraries contributed to more than 25.6 million raw small-RNA reads total, among which 23,468,606 (>91\% of the total) were adapter-trimmed, high-quality reads (qualified reads, Additional file 2: Table S1A). Among the qualified reads, $53.40 \%$ and $73.18 \%$ could map to the Cassava reference genome (http://www.phytozome.net) allowing zero and one mismatch (Additional file 2: Tables S1B and $\mathrm{S} 1 \mathrm{C}$ ), respectively, indicating a high sequencing quality despite that $\mathrm{SC} 124$ is different cultivar from the reference genome AM560. The qualified and genome mapped reads had lengths peaked at 21-nt and 24-nt, and carried twice more U's and A's than G's and C's as the first nucleotides (Additional file 1: Figures S2A and S2B). In comparison, the reads from miRNAs were dominantly 21-nt or 22-nt and carried preferentially U's at the first nucleotides (Additional file 1: Figures S2C and S2D).

To appreciate the potential regulatory effects of sncRNAs, four mRNA libraries, which were prepared using the plants from the same three chilling treatments and the normal condition, were sequenced individually using the Illumina RNA-seq protocol (see Methods, sequencing data in NCBI/GEO, accession \# GSE52178). Briefly, from more than $80 \%$ genome-mapped reads of more than 35.3 million raw reads, 12,689 (37.16\% of the 34,151 annotated Cassava mRNA genes), 16,023 (46.92\%), 15,144 (44.34\%) and 17,026 (49.85\%) mRNA genes were expressed under the NC, CA, CCA, and CS conditions, respectively (Additional file 2: Table S2, see Methods). Among the expressed genes were 2855, 1082 and 3297 differentially expressed genes in AC, CCA and $\mathrm{CS}$ in reference to $\mathrm{NC}$ (Additional file 2: Table S2). These differentially expressed genes were further analyzed, in addition to sncRNAs, in the study.

sncRNA species expressed in CCA treated and the normal castor bean plants were profiled following the same sequencing protocol as used for Cassava. The sequencing data, which have similar percentage of qualified reads and reads mapped to the reference genome and similar distributions of length and first nucleotide bias as those of Cassava, with one exception that miRNAs were dominantly 21-nt long in castor bean (Additional file 1: Figure S3 and Additional file 2: Table S3).

\section{Novel miRNAs in Euphorbia and their conservation Novel miRNAs}

Utilizing the sequencing data and a set of stringent criteria (see Methods), we identified 22 and 10 novel miRNAs in Cassava and castor bean, respectively (Table $1 \mathrm{~A}$ and B). Alignments of novel miRNA sequencing reads to the respective genomes are given in Additional files 3 and 4. If a newly detected miRNA has been reported in other plants, e.g., miR3627, the same family name was used; otherwise, a temporary new family name, e.g., novel-6, was introduced. The same temporary family names were used for Cassava and castor bean to indicate their conservation across the two species. For example, mes-novel-40 in Cassava (Table 1A) and rco-novel-40 in castor bean (Table 1B) belong to the same miRNA family. Among these novel miRNAs, four novel miRNAs in Cassava, novel-20, novel-52, novel-54, and novel-55, were highly represented as detected by sequencing reads (Table 1A). The sequence reads for these four miRNAs counted for $6 \%$ of the total reads from Cassava miRNAs.

\section{Re-annotation of known miRNAs}

We further analyzed the Cassava and castor bean miRNAs identified in our previous study [30] (miRBase version 20), which were detected by analyzing our deep sequencing data. Furthermore, 17 recently reported candidate miRNAs not in miRBase [31-33], which were named as reported- $k$ to distinguish them from the ones in miRBase, were also presented in our sequencing data (Additional file 2: Table S4). Among them, a newly reported candidate, reported-31 [31], counted for 138,426 of the total reads and ranked the 4-th most abundant among all miRNA families that were detected; it was only less abundant than $\mathrm{miR} 156 \mathrm{i} / \mathrm{j} / \mathrm{k}, \mathrm{miR} 166$ (all eight members), and miR167d/e/f (Additional file 2: Table S4). Besides, 163 individual miRNAs, belonging to 33 families in Cassava were listed in Additional file 2: Table S4. The miR156, miR171, miR166, and miR169 families constitute the largest miRNA families in Cassava (Table 2), with 11, 11, 11, and 29 members, respectively. All these miRNAs in Cassava and castor bean were re-annotated according to miRNA identification criteria (Additional file 2: Tables S4 and S5). Alignments of miRNA sequencing reads to the respective genomes are given in Additional files 5 and 6.

\section{Experimental validation and analysis of miRNAs}

We selected 95 known, newly reported and novel miRNAs to validate and assess miRNA's expression under four conditions in SC124 with qualitative real-time RT-PCR (qRTPCR) methods. As a result, 61 out of 95 miRNAs were detected in at least one of the four conditions, respectively (Figure 1A and Additional file 2: Table S6). Figure 1B shows examples of known and novel miRNAs by qRTPCR, and Figure 1C displays the detailed experimental results on novel miRNA novel-55 whose hairpin structure is shown in Figure 1D.

For known miRNAs, the detected miRNAs have a large number of reads (an average of 26,838 reads), but miRNAs that failed to be detected have smaller number of reads (an average of 2,869 reads). For example, two miRNA families (miR159 and miR167), which had more 
Table 1 Novel miRNAs in Cassava and castor bean identified from small-RNA profiling

\begin{tabular}{|c|c|c|c|c|c|c|c|c|c|}
\hline No. & Mature_sequence & \#. Reads & WGS_ID & Start & End & Strd. & Genomic region & Validation & Name \\
\hline \multicolumn{10}{|c|}{ (A) Twenty-two novel miRNAs were identified and experimental validated in Cassava } \\
\hline 1 & UGGACGCCAUUUUGACAGAUG & 248 & scaffold00847 & 1153336 & 1153495 & + & Intergenic & $\mathrm{N}$ & novel-3 \\
\hline 2 & CAAAUUAUAAUGGCAUUUUGA & 11 & scaffold02022 & 100612 & 100777 & + & Intergenic & nd & novel-10 \\
\hline 3 & UGGGUAAGUGGGGAAGAUAAC & 90 & scaffold02264 & 510281 & 510416 & + & Intergenic & $\mathrm{N}$ & novel-11 \\
\hline 4 & AAAUGGGACUCAUCAUAUGGUGGG & 50 & scaffold02658 & 994069 & 994318 & + & intron.2_Cassava4.1_019698m.g & Y & novel-14 \\
\hline 5 & UGGCCUAGAGUAGUGACCUCC & 346 & scaffold02936 & 84627 & 84693 & - & Intergenic & Y & novel-16 \\
\hline 6 & AGAUGGGUGGCUCGGGAAGAAG & 21209 & scaffold03604 & 533307 & 533466 & - & Intergenic & Y & novel-20 \\
\hline 7 & UUAUUUGAUCAAGGGAAAUUC & 154 & scaffold03802 & 905079 & 905143 & + & 3'UTR.1_Cassava4.1_011822m.g & N & novel-21 \\
\hline 8 & UUUGGGGUAAAUUUGGACCAAA & 46 & scaffold05335 & 14983 & 15180 & - & intron.4_Cassava4.1_025731m.g & N & novel-24 \\
\hline 9 & UGGCCUUUUGAGUUUGAGAAGACA & 100 & scaffold05694 & 371116 & 371230 & - & intron.6_Cassava4.1_022022m.g & $\mathrm{N}$ & novel-27 \\
\hline 10 & UUGGAAGAGCUUACUUUAAAU & 495 & scaffold05875 & 2354583 & 2354832 & - & Intergenic & N & novel-28 \\
\hline 11 & UCUGAAUCCCUGACGAAGCCU & 243 & scaffold05884 & 79762 & 79829 & + & Intergenic & Y & novel-29 \\
\hline 12 & UUUAUAUCAUGCAUAAUUAAG & 82 & scaffold05890 & 126 & 236 & - & Intergenic & N & novel-30 \\
\hline 13 & UGUCGCUGGAGAAAUGGCACUA & 80 & scaffold07240 & 11314 & 11420 & - & Intergenic & nd & novel-38 \\
\hline 14 & UUUUAAUGAUAGUAUAGGGGU & 12 & scaffold07290 & 137355 & 137549 & + & Intergenic & nd & novel-39 \\
\hline 15 & UGGGUGGGUGAGUGGAUAAGA & 172 & scaffold07996 & 160667 & 160818 & - & Intergenic & Y & novel-40 \\
\hline 16 & UCCAGGCAAGGAAAGCUUUUC & 28 & scaffold08542 & 27109 & 27229 & - & Intergenic & N & novel-44 \\
\hline 17 & UUGAGGGCUGUUUCCAGAAGC & 207 & scaffold10241 & 178034 & 178193 & + & Intergenic & Y & novel-50 \\
\hline 18 & UCUAUAUGGUCUGCGGUUACC & 219 & scaffold12301 & 237927 & 238086 & + & Intergenic & Y & novel-51-5p \\
\hline 18 & UGACCGCAGACCAUAUAGAAC & 446 & scaffold12301 & 237927 & 238086 & + & Intergenic & Y & novel-51 \\
\hline 19 & GGAAUGGGCGGUUUGGGAAAA & 29917 & scaffold04043 & 391309 & 391458 & - & Intergenic & Y & novel-52 \\
\hline 19 & UUCCCAAUGUCGCCCAUUCCGA & 890 & scaffold04043 & 391309 & 391458 & - & Intergenic & Y & novel-52-3p \\
\hline 20 & AAAAGGAAGAUGGAGGGCAUGA & 126 & scaffold03264 & 387326 & 387485 & - & Intergenic & nd & novel-53 \\
\hline 21 & ACUCUCCCUAAAGGCUUCAAC & 3851 & scaffold03581 & 762705 & 762813 & - & Intergenic & Y & novel-54 \\
\hline 22 & UAUGGGGGGAUUGGGCAAAAU & 38040 & scaffold03604 & 533082 & 533222 & - & intergenic & Y & novel-55-5p \\
\hline 22 & UUCCCAAGACCUCCCAUACCAG & 654 & scaffold03604 & 533082 & 533222 & - & intergenic & $\mathrm{Y}$ & novel-55-3p \\
\hline No. & Mature_sequence & \#. Read & WGS_ID & Start & End & Strd. & Genomic region & & Name \\
\hline \multicolumn{10}{|c|}{ (B) Ten novel miRNAs in castor bean } \\
\hline 1 & UGGGUGAGUGGAGAAGAUAAC & 14 & 30128 & 2013705 & 2013875 & - & intergenic & & novel-40 \\
\hline 2 & GGAAUGGGCGGUUUGGGAAAG & 3622 & 29586 & 144967 & 145126 & - & intergenic & & novel-52 \\
\hline 3 & ACUCUCUCUGAAGGCUUCAAA & 4755 & 29742 & 519324 & 519418 & - & intergenic & & novel-54 \\
\hline 4 & UAUGGGGGGAUCGGGCAAUAUU & 577 & 29660 & 174480 & 174647 & + & intergenic & & novel-55-5p \\
\hline 4 & UCUUCCCGAGACCUCCCAUACC & 281 & 29660 & 174480 & 174647 & + & intergenic & & novel-55-3p \\
\hline 5 & UCUUAUAGCAAUCAGGGGACUUG & 296 & 29877 & 66504 & 66639 & + & intergenic & & novel-63 \\
\hline 6 & UAGCAAAAGAUAGAACCGGAG & 225 & 29904 & 1112560 & 1112809 & + & intergenic & & novel-64 \\
\hline 7 & UCUGAUAGCAAAAGAUAGAAC & 528 & 29904 & 1112560 & 1112809 & - & intergenic & & novel-64as \\
\hline 8 & CGAGUCAUCUGACAGAAGUAG & 5102 & 29912 & 1877312 & 1877561 & + & intergenic & & novel-65 \\
\hline 9 & UGACGUGGCAUGAACUUCGGCA & 756 & 30074 & 662171 & 662379 & - & intron.6_30074.t000092 & & novel-66 \\
\hline 10 & UCCUCUGUCACAAAUGGCUUCCAG & 182 & 29912 & 1868939 & 1869188 & + & intergenic & & novel-67 \\
\hline
\end{tabular}

than $7 \mathrm{~K}$ reads, can also be detected in qRT-PCR validation (Figure 1B, Additional file 2: Tables S4 and S6). Similar to known miRNAs, the detected novel miRNAs had large numbers of reads (with an average of 28,501 reads), but undetected miRNAs typically had small numbers of reads (with an average of 137 reads). Mature 
Table 2 Conservation of Euphorbiaceous miRNA families across 9 plant species - M. esculenta (mes), $R$. communis (rco), P. trichocarpa(ptc), M. truncatula(mtr), G. max (gma), A. thaliana (ath), V. vinifera (vvi), O. sativa (osa) and $P$. patens(ppt)

\begin{tabular}{|c|c|c|c|c|c|c|c|c|c|}
\hline $\mathrm{miR}$ & mes & rco & ptc & mtr & gma & ath & vvi & osa & $\mathrm{ppt}$ \\
\hline 156 & 11 & 8 & 11 & 9 & 7 & 8 & 9 & 12 & 3 \\
\hline 159 & 4 & 1 & 6 & 1 & 4 & 3 & 3 & 6 & \\
\hline 160 & 8 & 3 & 8 & 5 & 1 & 3 & 5 & 6 & 9 \\
\hline 162 & 2 & 1 & 3 & 1 & 1 & 2 & 1 & 2 & \\
\hline 164 & 4 & 4 & 6 & 4 & 1 & 3 & 4 & 6 & \\
\hline 166 & 11 & 5 & 17 & 8 & 2 & 7 & 8 & 14 & 13 \\
\hline 167 & 9 & 3 & 8 & 1 & 7 & 4 & 5 & 10 & \\
\hline 168 & 1 & 1 & 2 & 1 & 1 & 2 & 1 & 2 & \\
\hline 169 & 29 & 3 & 32 & 17 & 5 & 14 & 25 & 17 & \\
\hline 171 & 11 & 7 & 14 & 7 & 3 & 3 & 9 & 9 & 2 \\
\hline 172 & 6 & 1 & 9 & 1 & 6 & 5 & 4 & 4 & \\
\hline 319 & 8 & 4 & 9 & 2 & 3 & 3 & 5 & 2 & 5 \\
\hline 390 & 3 & 2 & 4 & 1 & 2 & 2 & 1 & 1 & 3 \\
\hline 393 & 4 & 1 & 4 & 2 & 1 & 2 & 2 & 2 & \\
\hline 394 & 3 & 2 & 2 & & 2 & 2 & 3 & 1 & \\
\hline 395 & 5 & 5 & 10 & 18 & 3 & 6 & 14 & 25 & 1 \\
\hline 396 & 6 & 1 & 7 & 2 & 5 & 2 & 4 & 9 & \\
\hline 397 & 2 & 1 & 3 & & 2 & 2 & 1 & 2 & \\
\hline 398 & 1 & 2 & 3 & 3 & 2 & 3 & 3 & 2 & \\
\hline 399 & 7 & 6 & 12 & 17 & & 6 & 9 & 11 & \\
\hline 403 & 2 & 2 & 3 & & 2 & 1 & 6 & & \\
\hline 408 & 2 & 1 & 1 & 1 & 4 & 1 & 1 & 1 & 2 \\
\hline 477 & 9 & 1 & 2 & & & & 1 & & 8 \\
\hline 482 & 1 & & 4 & 1 & 5 & & 1 & & \\
\hline 530 & 2 & 1 & 2 & & 2 & & & 1 & \\
\hline 535 & 3 & 1 & & & & & 3 & 1 & 4 \\
\hline 827 & 1 & 1 & 1 & & & 1 & & 3 & \\
\hline 828 & 2 & 2 & 2 & & 2 & 1 & 2 & & \\
\hline 1446 & 1 & 1 & 5 & & & & & & \\
\hline 2111 & 2 & 1 & & 19 & 1 & 2 & 1 & & \\
\hline 2275 & 1 & & & & & & & 1 & \\
\hline 2950 & 1 & & & & & & 1 & & \\
\hline 3627 & 1 & 2 & 2 & & & & 1 & & \\
\hline
\end{tabular}

Listed in the tables are the numbers of individual members of miRNA families.

miRNAs may arise to an abundant level from both arms. For example, four miRNAs (novel-51, novel-52, novel-54 and novel-55), each of which had more than 200 reads, had their mature miRNAs detected by qRTPCR (Additional file 2: Table S6). Taken together, miRNAs with low numbers of sequencing reads were likely not to be detected by qRT-PCR, reflecting that deep sequencing was more sensitive to low abundant transcripts.

\section{Conservation of miRNAs}

The conservation of all known, newly identified and novel miRNAs in Cassava and castor bean was examined in reference to seven diverse plant species, i.e., Populus, Medicago, soy bean, Arabidopsis, grapes, rice and moss (Physcomitrella patens) (Table 2). Eight known miRNA families - miR156, miR160, miR166, miR171, miR319, miR390, miR395, and miR408 - were evolutionally conserved across the 9 species (Table 2). Another 10 annotated miRNA families - miR159, miR162, miR164, miR167, miR168, miR169, miR172, miR393, miR396, and miR398 - were also conserved in at least eight species (Table 2). The highly expressed report-31 had no homologs in castor bean and the other seven plants. The conservation of these miRNAs is broad, ranging from the angiosperm lineages to vascular plants, suggesting their essential functions in plants [15].

Remarkably, most novel miRNA families identified in the current study were Euphorbia specific. In these miRNA families, five (novel-14, novel-40, novel-52, novel-54, and novel-55) exist in both Cassava and castor bean, while 18 and 6 novel miRNAs were Cassava and castor bean specific, respectively (Table 1 ). Among the highly expressed novel miRNAs in Cassava (Table 1A), three (novel-52, novel-54, and novel-55) had homologs in castor bean, though they did not appear in other plants beyond Euphorbia. The existence of lineage/species specific miRNAs in Cassava and castor bean indicated some specific roles that these miRNAs may play in stress response in Euphorbiaceous plants.

\section{MicroRNA-triggered secondary siRNAs}

A genome-wide search of small RNAs resembling secondary siRNAs (see Methods) resulted in 26 and 21 genomic loci enriched with 21-nt long in length in the small-RNA libraries of Cassava and castor bean, respectively (Table 3, Additional file 2: Tables S7 and S8). These genomic loci were targeted by 17 miRNA families in Cassava (Additional file 2: Table S7) and 12 miRNA families in castor bean (Additional file 2: Table S8). The majority (85\%) of these siRNA-generating genomic loci in Cassava correspond to protein-coding genes (Table 3). These genes fell into several classes. The first was the set of three loci corresponding to the TAS3 genes, known hosts of ta-siRNAs, despite that only one TAS3 locus has been annotated in the current Cassava reference genome [13]. The second and biggest class consisted of 10 NB-ARC domain-containing disease resistance genes, which are receptors sensing intracellular perturbations [34]. The third class contained 2 auxin response factors and 2 auxin signaling F-box genes.

Many of these siRNA-generating loci and their targeting miRNA candidates were conserved in castor bean (Table 3). As expected, the three TAS3 loci and the 


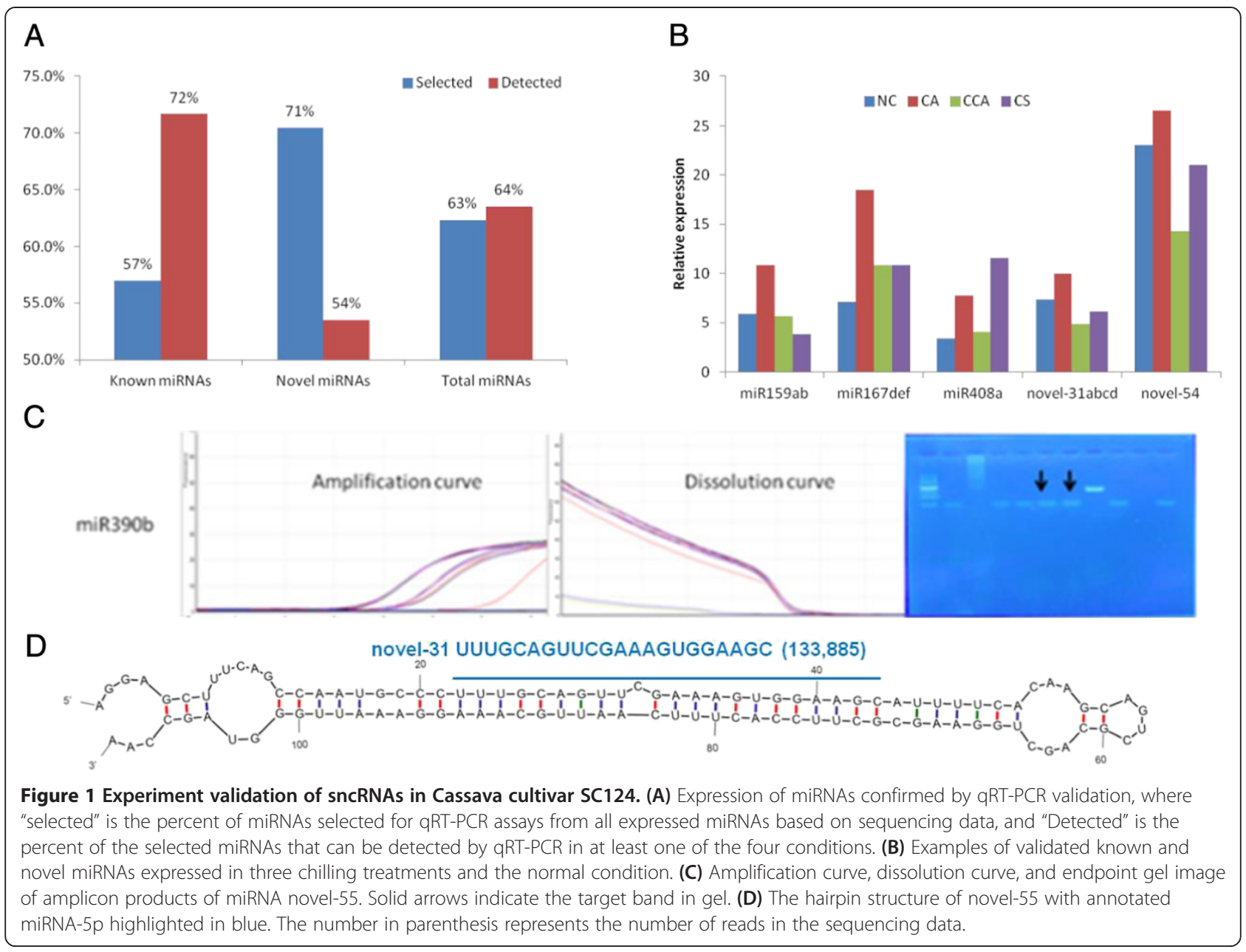

targeting miRNA (i.e., miR390), 3 auxin response factors, and auxin signaling F-box genes were targeted by miR167 and miR393 and two GRAS family transcription factors were targets of miR171. Such a strong conservation suggested some common regulatory functions of those siRNA-yielding loci in Euphorbiaceous plants.

\section{ta-siRNAs triggered by miR390 and Novel TAS3 genes}

A genome-wide homologue search of TAS genes in Euphorbia concluded that TAS1 and TAS2 genes did not exist in the Cassava and castor bean genomes. This is consistent with the fact that miR173, which triggers ta-siRNAs in homologies of TAS1 and TAS2 in Arabidopsis, is not conserved in Euphorbia [15]. TAS4 did not seem to appear in Cassava or castor bean either, although miR828, the initiator of TAS4siRNAs in Arabidopsis, exists in Cassava and castor bean [15]. One TAS3 gene is currently annotated in the Cassava reference genome (gene ID: $019138 \mathrm{~m}$, [13]). This is in sharp contrast with three TAS3 genes in Arabidopsis $($ AtTAS3a/b/c) [16] and four TAS3 genes in $P$. patens $(P p T A S 3 a / b / c / d)$ [13].
We identified two novel TAS3 loci in both Cassava and castor bean genomes by searching for a pair of miR390 target sites (Figures 2, Additional file 1: Figures S4, S5 and S6). The currently annotated TAS3 gene was thus named as TAS3 $a$ and the two new TAS3 genes as TAS3b (Additional file 1: Figures S4 and S5) and TAS3c (Figure 2 and Additional file 1: Figure S6). Importantly, a substantial number of sequencing reads, arranged in phasing, appeared in the regions defined by the two miR390 target sites (Figure 2A, Additional file 1: Figures S4A, S5A and S6A). Following the standard nomenclature [35], the register of phased siRNAs was named as D1, D2, and so on, starting from the $5^{\prime}$-end miRNA target site, and the orientation was indicated by suffix "+" for the positive (or the original transcript) strand, or "-" for the negative (or the RdRP synthesized) strand.

The two binding sites of miR390 on TAS3c gene have a highly complementary sequence match (Figure 2B, left panel). Moreover, nearly perfect matches around the 10-nt from the $5^{\prime}$-end of miR390 binding sites suggested that both binding sites were cleaved, which were further supported by the phased sequencing reads starting 
Table 3 Distribution of genes that are targeted by miRNAs and subsequently produce candidate secondary siRNAs in (A) Cassava and (B) Castor bean, and their function categories

\begin{tabular}{|c|c|}
\hline \#. Genes & Transcripts yielding 21-nt enriched siRNAs \\
\hline \multicolumn{2}{|c|}{ A. Cassava } \\
\hline 2 & $\begin{array}{l}\text { ATPase E1-E2 type family protein/haloacid } \\
\text { dehalogenase-like hydrolase family protein }\end{array}$ \\
\hline 2 & auxin response factor 8 \\
\hline 2 & auxin signaling F-box 3 \\
\hline 1 & dicer-like 1 \\
\hline 2 & F-box/RNI-like superfamily protein \\
\hline 1 & GRAS family transcription factor \\
\hline 10 & NB-ARC domain-containing disease resistance protein \\
\hline 1 & phosphate transporter $1 ; 3$ \\
\hline 1 & scarecrow-like 3 \\
\hline 4 & TAS3 and unannotated loci \\
\hline \multicolumn{2}{|c|}{ B. Castor bean } \\
\hline 3 & auxin response factor \\
\hline 1 & auxin signaling F-box \\
\hline 1 & $\begin{array}{l}\text { basic helix-loop-helix (bHLH) DNA-binding superfamily } \\
\text { protein }\end{array}$ \\
\hline 1 & Cupredoxin superfamily protein \\
\hline 1 & disease resistance protein (TIR-NBS-LRR class), putative \\
\hline 1 & Galactose oxidase/kelch repeat superfamily protein \\
\hline 2 & GRAS family transcription factor \\
\hline 1 & Integrase-type DNA-binding superfamily protein \\
\hline 1 & Leucine-rich receptor-like protein kinase family protein \\
\hline 1 & Malectin/receptor-like protein kinase family protein \\
\hline 1 & SET domain protein \\
\hline 3 & squamosa promoter binding protein-like \\
\hline 1 & TCP family transcription factor \\
\hline 3 & TAS3 \\
\hline
\end{tabular}

at the position of the cleavage sites of both $5^{\prime}$ - and $3^{\prime}$-end target sites (Figure 2B, right panel). These two-cleavage sites have been reported for TAS3 genes in P. patens [13] but different from that of Arabidopsis TAS3a where only the 3 '-end target site is cleaved [7]. Similar cleavage sites of miR390 on the TAS3c gene appeared in castor bean (Additional file 1: Figures S6D and S6E).

Interestingly, the regions between the two pairs of miR390 target sites on TAS3c in the two Euphorbiaceous species had the same length of 190-nt and shared a high sequence identity (Figure 2C). The regions around the two target sites had almost identical sequences, suggesting a strong conservation on and near the miR390 target sites. Importantly, siRNA from D4+ register (Figure 2A) was highly conserved with tasiARF, a well-studied tasiRNA targeting an ARF gene in Arabidopsis (ASRP2141)
[7]. It is noteworthy that TAS3a and TAS3b also host tasiARF-like siRNAs in Euphorbia. However, sequence alignment revealed that there existed three distinct tasiARF-like siRNA species encoded by TAS3a/b/c; a slight sequence difference near the $3{ }^{\prime}$-ends existed in the three tasiARFs (Additional file 1: Figure S7). Finally, the three TAS3 genes encoded different numbers of tasiARF. TAS3a and TAS3b maintained two tasiARFs adjacent to each other (e.g., tasiARF.1 and tasiARF.2 in Additional file 1: Figures S4A and S5A), while TAS3c contained a single tasiARF (Figure 2A).

A total of 8,479 reads from the small-RNA libraries of Cassava were mapped to the TAS3c region, among which $85.1 \%$ were 21 -nt long. Among these small RNAs, $5,884(66.4 \%$ of the total of 8,479$)$ were arranged in phase with reference to the putative cleavage site at either the $5^{\prime}$ or $3^{\prime}$ target site of miR390. Interestingly, $20.5 \%$ of 21-nt small RNAs have the first nucleotides at the positions shifted by $\sim 10$ - or 11-nt from the majority phased small RNAs. These shifted siRNAs followed the phasing register set by siRNA D8-, indicating that siRNAs arising from D8- could target and cleave the original transcripts, setting a secondary phasing register different from the register set by miR390. The most prominent example of such shifted siRNAs was located at the middle of D5+ and D3- (Figure 2A). Similarly, 2,195 sequencing reads, $81 \%$ of which were 21 -nt long, were derived from TAS3c in castor bean. Secondary, shifted siRNAs (21\% of the total siRNAs from TAS3c) were also observed (Additional file 1: Figure S6C), similar to that in Cassava TAS3c, exemplified by the peaks in the middle of D6+, D6- and D5-. Taken together, TAS3c in Cassava and castor bean produced ample ta-siRNAs, majority of which were arranged in 21-nt phasing, while a small amount of which had a register shifted half way from the main register.

\section{Candidate secondary siRNAs triggered by miRNAs}

A total of 77 and 86 NBS-LRR genes are annotated in the current Cassava and castor bean genomes, respectively [36]. However, only one castor bean NBS-LRR genes was predicted to be targeted by miR396 to produce enriched siRNAs of 21-nt long (Table 3). This is in contrast to the results in Medicago and tomatoes, where a large number of NBS-LRR genes have been reported to be targeted by one or two miRNA initiators, such as miR482 $[18,19]$. We were able to identify one locus encoding miR482 in Cassava genome (Additional files 5). However, miR482 in Cassava was rarely sequenced in the current data sets (Additional file 5), indicating that miR482 might not be expressed in the tissues we examined. Alternatively, it is plausible that miR482 did not regulate NBS-LRR genes in Euphorbiaceous species, which is consistent with the prediction that few NBS-LRR 


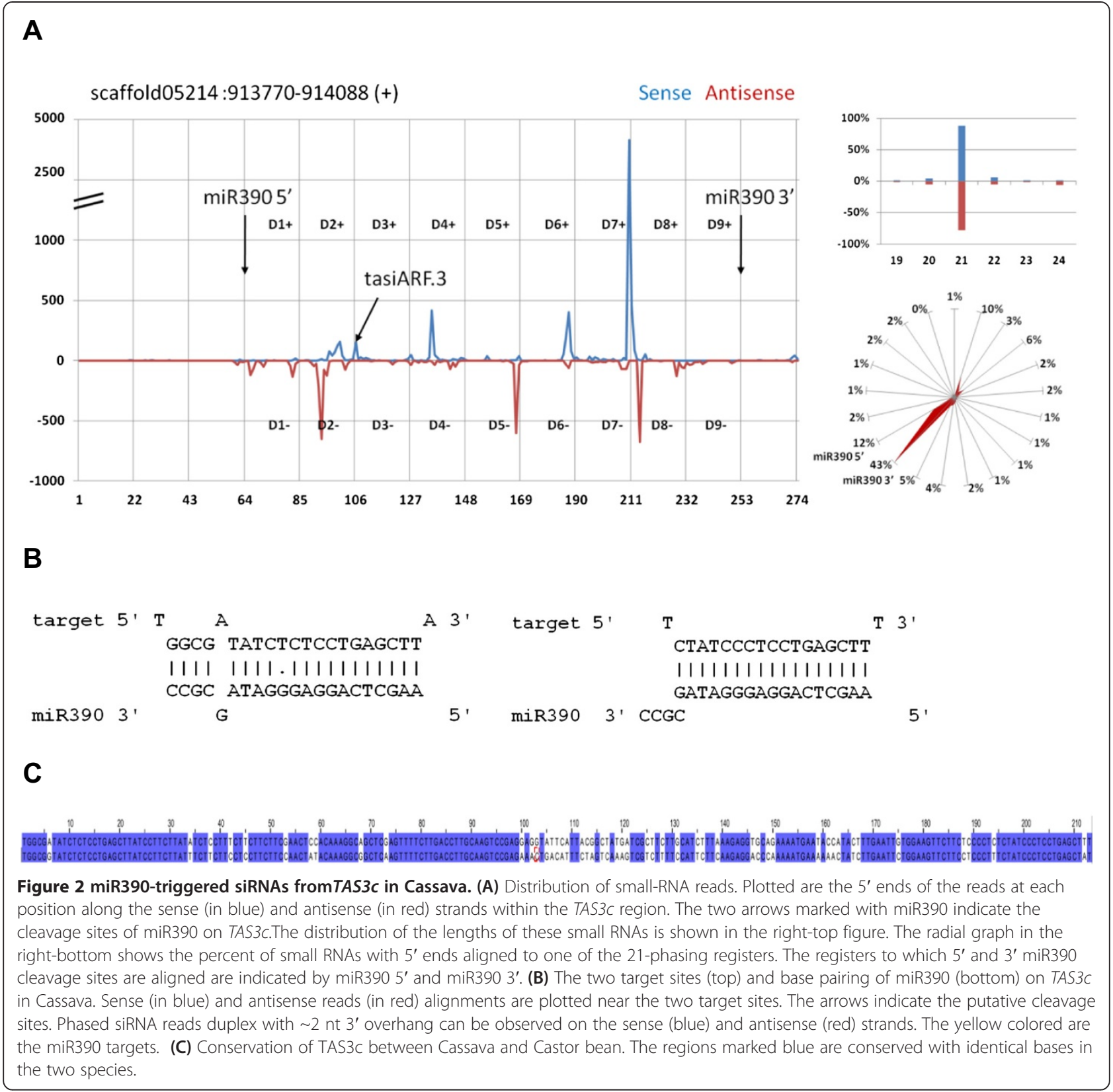

genes were targeted by miR482 in Cassava or castor bean, respectively.

ta-siRNAs at TAS3 loci were found to be arranged in phasing. Nevertheless, many other siRNAs were not arranged in a precise 21-nt phasing pattern (Additional file 2: Table S7). For example, an unannotated genomic locus associated with report-31 produced 14,369 siRNA reads in total, $84 \%$ of which were 21 -nt long (Additional file 1: Figures S8A and S8B), but interestingly, the siRNAs were not arranged in phasing (Additional file 1: Figures S8C), different from the phasing pattern of siRNAs from the TAS3 loci. Non-phasing ta-siRNAs have also been observed in Arabidopsis, where RDR6dependent siRNAs derived from miRNA target loci (e.g. miR168 at AGO1, miR472 at several CC-NBS-LRR genes) were found not to be arranged in a phasing pattern either [16]. Therefore, we reported both phased and non-phased siRNA candidates from miRNA-targeted genes.

\section{Experimental validation of secondary siRNAs and their targets}

We experimentally validated three ta-siRNAs including tasiARF1/2 from TAS3a/b genes and the highly sequenced D8+ siRNA from the newly identified TAS3c 
gene (Additional file 1: Figure S9). The qRT-PCR result showed consistent amplification and sharp dissolution curves as well as clear bands of three siRNAs, supporting the genuine presence of the three ta-siRNAs (Additional file 1: Figure S9).

Further, to investigate the effect of sncRNAs on target genes, we used 5 '-RACE to assess the cleavage activities of miRNAs and secondary siRNAs of TAS3 on their targets. The miRNAs that we tested could indeed cleave their targets, as shown by the cleavage sites of miR167 on ARF8 and that of miR393 on ABF3 (Table 4). The two putative cleavage sites of miR390 on TAS3c were cleaved (Table 4), in agreement with the reads from the RNA-seq data (Figure 2B). Moreover, ta-siRNAs of TAS3 were also able to cleave their target genes. The subsequent secondary ta-siRNAs - tasiARF1, tasiARF2, and tasiARF3 - cleaved their respective targets; specifically, tasiARF1 cleaved both ARF3 and ARF4, tasiARF2 cleaved NAC2 and tasiARF3 cleaved ARF3 (Table 4 and Figure 3). Interestingly, tasiARF1 and tasiARF3 degraded the same target (AFR3, $002399 \mathrm{~m}$ ) at the same cleavage site (the 11-th nucleotide from the $5^{\prime}$ end of the small-RNA binding site) with different cleavage efficiency (i.e., approximately by a 6 to 1 ratio) under the CS condition (Table 4, Figure $3 \mathrm{~A}$ and $3 \mathrm{~B}$ ). Moreover, one putative secondary siRNA derived from report-31 initiated transcript, i.e., siReport-31, was able to cleave its target gene, CUL4. Note that some cleavage sites detected by 5 '-RACE were separated by consecutive segments of 21-nt long, e.g., between the cleavages sites at 21-nt and 84-nt downstream from the tasiARF1 cleavage sites on ARF4 (data not shown).

\section{Candidate siRNAs originated from natural antisense transcripts}

Following our method for identifying cis-NATs and using the threshold of 25-nt overlapping [26], 135 and 63 pairs of cis-NATs were identified, respectively, in the Cassava and castor bean reference genomes (Additional file 2: Tables S9 and S10). Among the 135 cis-NATs in Cassava, 48 (36\%), 15 (11\%) and $72(53 \%)$ pairs were arranged in the convergent ( $3^{\prime}-3^{\prime}$ overlap), divergent ( $5^{\prime}-5^{\prime}$ overlap) and enclosed orientations, respectively (Additional file 2: Tables S9). Note that the percentage of enclosed cis-NATs was greater than that in Arabidopsis $[25,26]$. In castor bean, the same number of 23 cis-NAT pairs appeared in the enclosed and divergent categories and 17 were convergent (Additional file 2: Table S10).

Seven and 1 cis-NAT in Cassava and castor bean, respectively, were detected to yield nat-siRNAs, each of which gave rise to at least 10 reads in the overlapping region (Table 5). One example of cis-nat-siRNAs was from the cis-NAT pair of two genes, $013132 \mathrm{~m}$ and $020539 \mathrm{~m}$ on scaffold07238 (cis-NAT-1 in Table 5), arranged in a convergent fashion (Figure 4); a total of 271 reads aligned to the overlap region of the two genes. Approximately $80 \%$ of the mappable reads appeared in the cis-NAT overlapping region, showing that cis-natsiRNAs were predominantly derived from the overlapping region. Transcription of the two NAT genes were confirmed by Expressed Sequence Tags (ESTs; accession number asmbl_5742 and _5743) and directly supported by our RNA-seq data of the Cassava transcriptome profiling under the four conditions (Additional file 2: Table S11).

Table 4 miRNAs and siRNAs cleavage sites detected by 5'RACE

\begin{tabular}{|c|c|c|c|c|c|}
\hline Genes & Condition & Basepair & Target ID & & Annotation \\
\hline \multirow{2}{*}{ miR390-TAS3c binding site \#1 } & $C A+C C A$ & $1(1)$ & & & \\
\hline & CS & $11(2), 1(6), 0(1)$ & craffeld05211 & $T \Delta c_{3}$ & Non-coding gene, the inter-space between NO.1 and \\
\hline \multirow{2}{*}{ miR390-TAS3c binding site \#2 } & $C A+C C A$ & $5(1)$ & 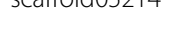 & 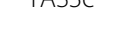 & NO.2 basepair match is $168 \mathrm{bp}$. \\
\hline & CS & $5(1)$ & & & \\
\hline \multirow[t]{2}{*}{ miR167-ARF8 } & $C A+C C A$ & $9(1), 7(1)$ & $001923 \mathrm{~m}$ & ARF8 & auxin response factor 8 \\
\hline & NC & $10(1), 17(1)$ & & & \\
\hline \multirow[t]{2}{*}{ miR393-ABF3 } & $C A+C C A$ & $11(8)$ & 004520 m & AFB3 & auxin signaling F-box 3 \\
\hline & CS & $11(7)$ & & & \\
\hline \multirow{2}{*}{ tasiAFR3-ARF3 } & $C A+C C A$ & $11(1)$ & & & \multirow{3}{*}{$\begin{array}{l}\text { Transcriptional factor B3 family protein/auxin-responsive } \\
\text { factor AUX/IAA-related }\end{array}$} \\
\hline & CS & $11(6)$ & $002399 \mathrm{~m}$ & ETT/ARF3 & \\
\hline tasiARF1-ARF3 & CS & $11(1)$ & 002399 m & ETT/ARF3 & \\
\hline \multirow{2}{*}{ tasiARF1-ARF4 } & $C A+C C A$ & $10(1)$ & \multirow{2}{*}{001979 m } & \multirow{2}{*}{ ARF4 } & \multirow{2}{*}{ auxin response factor 4} \\
\hline & CS & $10(1)$ & & & \\
\hline tasiARF2-NAC2 & CS & $11(2)$ & $027253 \mathrm{~m}$ & NAC2 & NAC domain containing protein 2 \\
\hline siReport31-CUL4 & $C A+C C A$ & $18(1)$ & $001768 \mathrm{~m}$ & CUL4 & cullin4 \\
\hline
\end{tabular}

Each target site was detected by ten clones. "Basepair" column lists the specific cleavage site from the 5' end of a miRNA or siRNA and the number of clones (in parenthesis) that were detected to have cleavage products. 


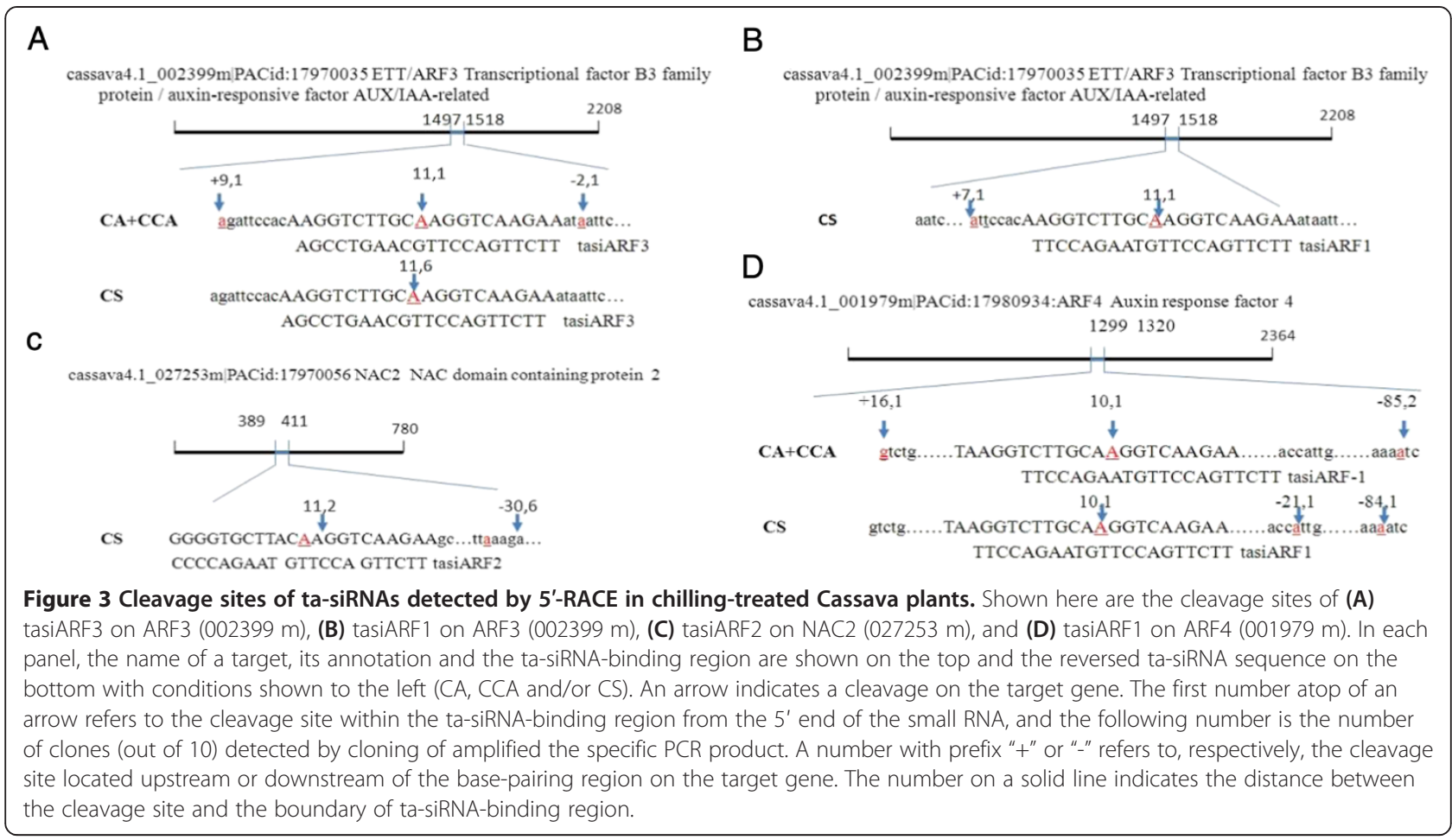

Unlike miRNAs, many of which are highly conserved in plants [15], the majority plant cis-nat-siRNAs are not conserved [26]. Interestingly, one cis-NAT pair detected in Cassava (013132 m and $020539 \mathrm{~m}$ ) was conserved in Arabidopsis (AT1G01720/AT1G01725; Additional file 1: Figure S10A), which overlap by $\sim 700$-nt in the $3^{\prime}-3^{\prime}$ orientation based on the Arabidopsis gene and EST annotation (red box in Additional file 1: Figure S10A). A few small RNAs, $85 \%$ of which were 21 -nt long, arise from the cis-NAT region in chilling-stressed Arabidopsis shoots [26]. In addition, castor bean genome appeared to have one homologous gene of AT1G01720 but no homologous gene for AT1G01725 that was essential for generating cis-NAT1 (Additional file 1: Figure S10B).

\section{Perturbed sncRNAs in response to chilling stress and acclimation}

A substantial number of miRNAs were DE under the chilling stresses with respect to the normal control and were $\mathrm{DE}$ across different stress conditions. Among the 150 (111 known and 39 novel and newly detected) miRNAs expressed in Cassava, 114 (78.6\% of the total) had at least

Table 5 cis-NAT pairs that generate siRNAs in Cassava and castor bean

\begin{tabular}{|c|c|c|c|c|c|c|c|c|}
\hline \multirow[b]{2}{*}{ ID } & \multirow[b]{2}{*}{ Scaffold } & \multirow[b]{2}{*}{ Type } & \multirow[b]{2}{*}{ Start } & \multirow[b]{2}{*}{ End } & \multicolumn{2}{|c|}{+ Strand } & \multicolumn{2}{|c|}{ - Strand } \\
\hline & & & & & \# Reads in $\mathrm{OL}^{\mathrm{a}}$ & $21 \mathrm{nt}^{\mathrm{b}}$ & \# Reads in $\mathrm{OL}^{\mathrm{a}}$ & $21 \mathrm{nt} \%^{\mathrm{b}}$ \\
\hline \multicolumn{9}{|l|}{ Cassava } \\
\hline cis-NAT-1 & scaffold07238 & $3^{\prime}-3^{\prime}$ & 376957 & 377910 & 40 & $85.0 \%$ & 175 & $66.3 \%$ \\
\hline cis-NAT-2 & scaffold11378 & $3^{\prime}-3^{\prime}$ & 285315 & 286689 & 53 & $57.2 \%$ & 10 & $60.0 \%$ \\
\hline cis-NAT-3 & scaffold08316 & $3^{\prime}-3^{\prime}$ & 598264 & 598958 & 33 & $60.6 \%$ & 7 & $100.0 \%$ \\
\hline cis-NAT-4 & scaffold12317 & $3^{\prime}-3^{\prime}$ & 65657 & 66262 & 26 & $65.4 \%$ & 6 & $83.3 \%$ \\
\hline cis-NAT-5 & scaffold03802 & $3^{\prime}-3^{\prime}$ & 435292 & 436201 & 4 & $100.0 \%$ & 24 & $54.2 \%$ \\
\hline cis-NAT-6 & scaffold11581 & $3^{\prime}-3^{\prime}$ & 786965 & 787920 & 7 & $85.7 \%$ & 15 & $53.3 \%$ \\
\hline cis-NAT-7 & scaffold06688 & $3^{\prime}-3^{\prime}$ & 321683 & 322092 & 8 & $87.5 \%$ & 7 & $85.7 \%$ \\
\hline \multicolumn{9}{|c|}{ Castor bean } \\
\hline Cis-NAT-8 & 29701 & $5^{\prime}-5^{\prime}$ & 185445 & 186292 & 112 & $94.6 \%$ & 152 & $94.7 \%$ \\
\hline
\end{tabular}

${ }^{\mathrm{a}} \mathrm{OL}$ stands for overlapping regions.

${ }^{\mathrm{b}} \mathrm{Abundance}$ of 21-nt reads expressed as the percentage of the total reads. 


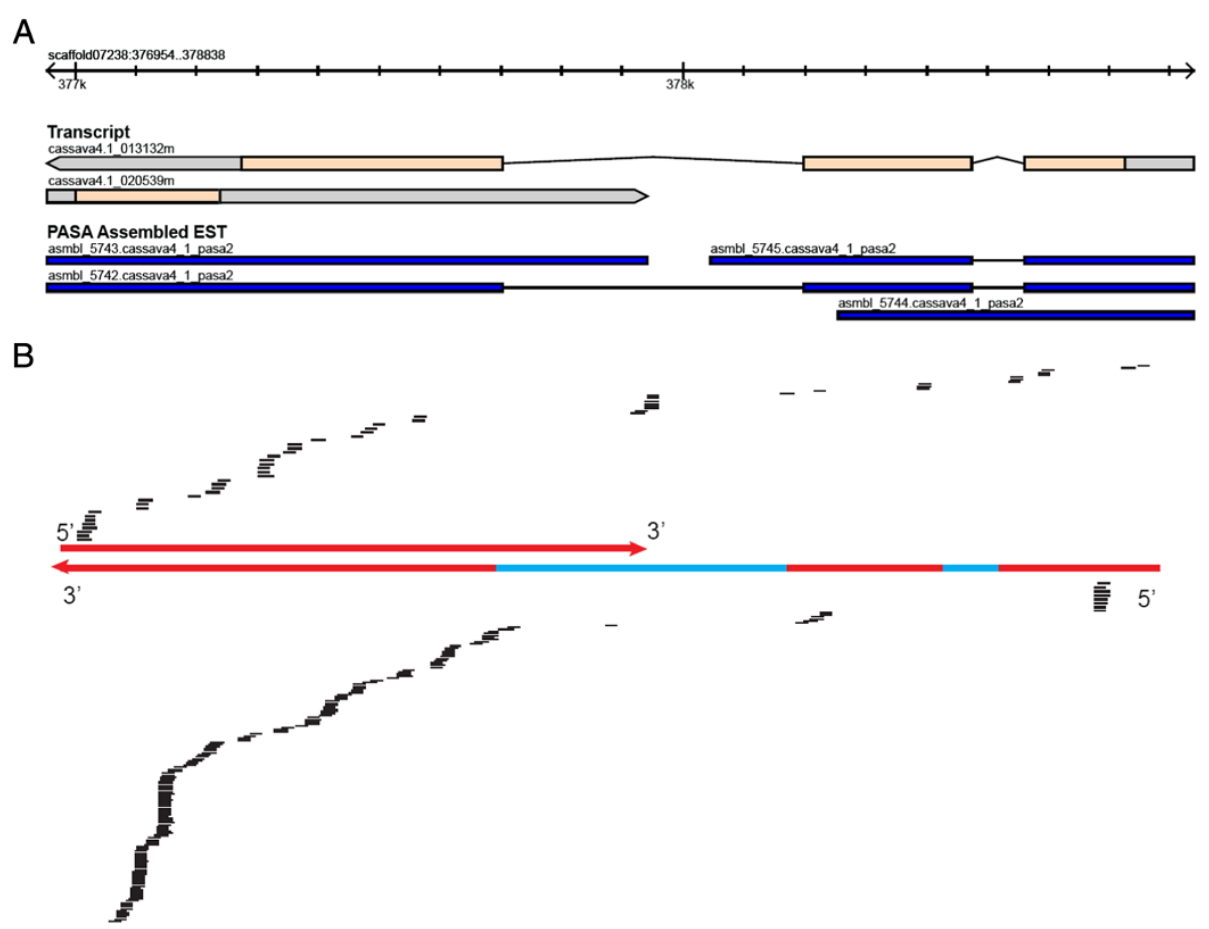

Figure 4 A cis-NAT candidate locus yields 21-nt enriched siRNAs. (A) cis-NAT pair of no apical meristem gene (NAM, $013132 \mathrm{~m}$ ) and unannotated gene $(020539 \mathrm{~m})$ in Cassava. The EST track displays the EST sequences from the Phytozome database [36]. (B) Small RNAs from the positive and negative strands are displayed above and below the NAT pairs, respectively. The red and blue regions on the gene model represent exons and introns, respectively.

1.5 -fold change under one of the six comparisons (Additional file 2: Table S12A). Fifteen DE miRNAs were further experimentally analyzed (Figure $5 \mathrm{~A})$. Note that the miR319 and miR395 families are well conserved in plants, and miR477 is conserved in Populus, Arabidopsis, Grape vine and Moss (Table 2A), suggesting their conserved function in plant stress response. As for the novel chilling-responsive, highly DE Cassava miRNAs, three (novel-51, -52 and novel-55-5p) were up-regulated and one (novel-16) were down-regulated across the chilling treatments. Three novel miRNAs (novel-20, novel-54-5p and novel-55-3p) were not differentially expressed in the three chilling treatments in SC124 cultivar. In contrast, the four miRNAs were all highly up-regulated at $4^{\circ} \mathrm{C}$ under both CCA and CS of the C4 cultivar (data not shown), a more chilling tolerance cultivar. Consistently, the expression patterns of novel-52 and novel-55-5p were significantly differentially expressed in the three chilling stresses with respect to the control condition, i.e., one was up-regulated and one down-regulated in the CA condition, while both were up-regulated under the CCA and CS conditions (Figure 5B).

The 26 putative siRNA-yielding loci in Cassava were further assessed across six comparisons for their potential for producing differentially expressed siRNAs. In the loci analyzed, $12(46 \%)$ of the 26 loci exhibited differential expression with at least 2-fold change under at least one of the six comparisons (Additional file 2: Table S12B). The miRNAs that initiated siRNAs were highly induced in all three chilling stress conditions compared to NC (Figure 5C). miR167, miR390 and miR393 were also differentially expressed under chilling stress; miR167 was up-regulated under CA while miR393 was up-regulated under CCA in reference to the normal condition. Some of the ta-siRNAs from TAS3 were validated under the four chilling treatments using qRT-PCR. tasiARF-2 and $\mathrm{D} 8+$ were down-regulated under the CCA vs NC condition (Figure 5C), indicating that special function of the ta-siRNAs might take part when Cassava was transferred from $14^{\circ} \mathrm{C}$ to $4^{\circ} \mathrm{C}$.

The targets of differentially expressed miRNAs and siRNAs are of special interest, particularly those that have expression patterns that are anti-correlated with that of their regulatory, differentially expressed siRNAs because such relationships provide information of siRNAs' potential regulatory function. A total of 21 putative targets were recognized to have expression patterns that are anticorrelated with that of differentially expressed targeting siRNAs in the six comparisons considered (Additional file 1: Figure S11). Among the anti-correlated pairs of siRNAs and their targets, many were related to genes involved in chilling-stress and chilling-acclimated response. 
A

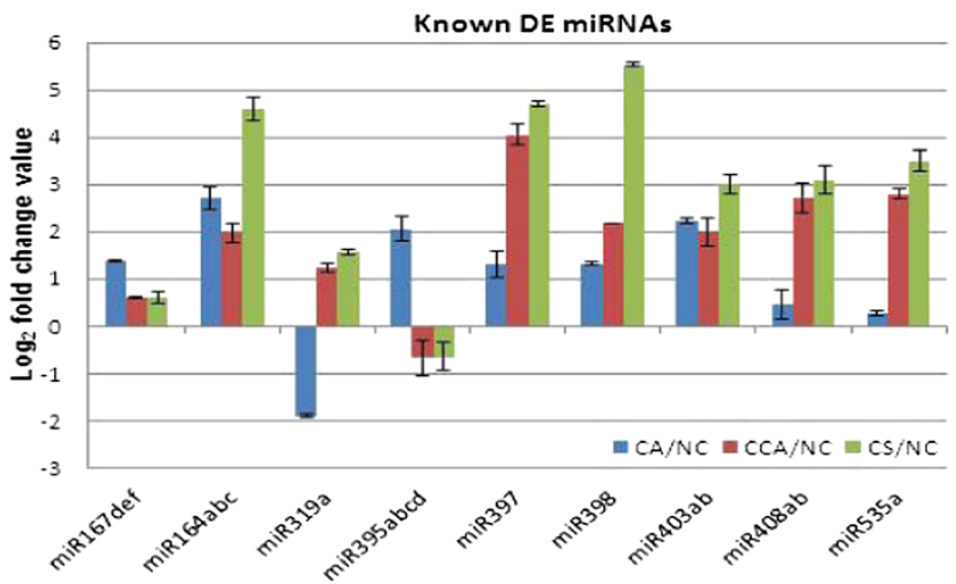

B

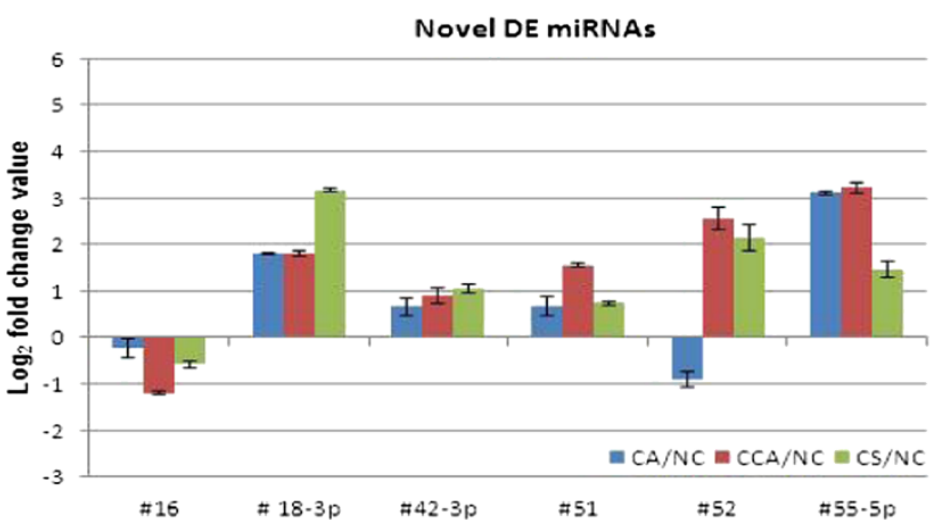

C

TasiRNAs and initiated miRNAs

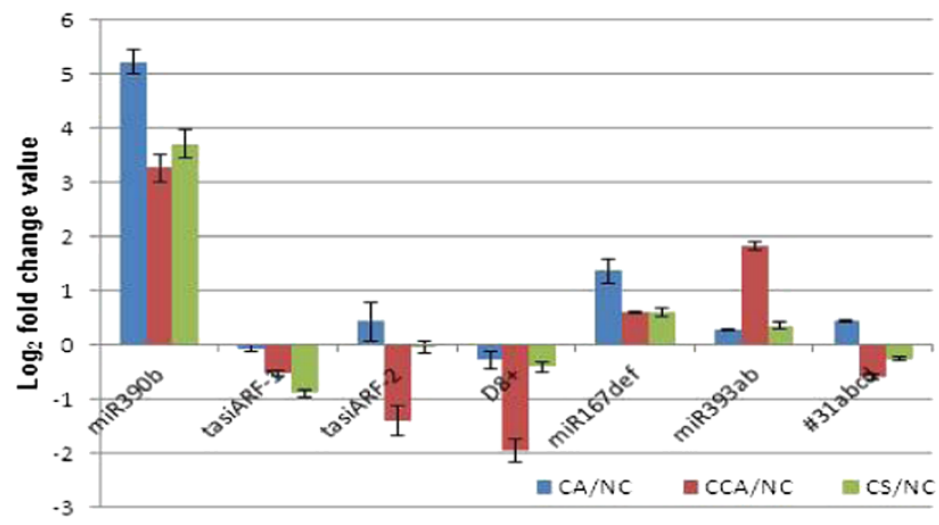

Figure 5 Experimental validation of differential expression of (A) known miRNAs, (B) novel miRNAs and (C) ta-siRNAs in three chilling stress treatments, CA, CCA and CS, with respect to the normal condition in Cassava SC124 cultivar.

For example, siRNAs derived from the auxin signaling F-box 3 gene $(A F B 3,004514 \mathrm{~m})$ was down-regulated in the CA condition with respect to $\mathrm{NC}$ and the host geneAFB3 was also down-regulated in CA vs. NC conditions, as determined by RNA-seq signals. It has been reported that F-Box protein-encoding genes respond to chilling stress in rice [37], leading to changes in gene expression. More importantly, siRNAs from $A F B 3$ were found to target an amino acid permease gene $(005642 \mathrm{~m})$ that was highly up-regulated ( $>25$ fold) in the CA vs NC condition based on RNA-seq profiling. The amino acid permease gene, which functions in amino acid translocation, has been found to be up-regulated in leaves of Citrus unshiu when it is fully acclimated after exposure to the chilling. The up-regulated level of the amino acid permease in C. unshiu indicates that active transport and 
metabolism of amino acids are necessary under low temperature conditions, and the roles of the protein has been found to be related to chilling acclimation [38].

While cis-nat-siRNAs from one strand are expected to regulate the gene on the antisense strand of a NAT pair, the differential expression of cis-nat-siRNAs may lead to the anti-correlated expression of their targets $[20,23,25]$. Two of the seven cis-nat-siRNAs were differentially expressed with at least 2-fold changes in at least one of the six comparisons that we considered (Additional file 2: Table S12C). One of these cis-NATs was a gene $(013132 \mathrm{~m})$ that was annotated as a NAC domain transcriptional regulator superfamily protein. NAC proteins have been implicated to participate in a wide range of plant developmental processes. Numerous NAC domain proteins have also been implicated in plant abiotic stress responses such as drought and chilling shock response [39].

\section{Discussion}

Plant miRNAs are associated with a variety of biological processes including development and stress responses [4]. Cold inducible miRNAs have been recently studied in variety of plant model species including $A$. thaliana, $O$. sativa (rice) and T. aestivum (wheat) [40-43]. The results obtained so far showed that miRNAs play important roles in regulating gene expression in response to chilling stress. The results from the current deepsequencing based study of two Euphorbia's species further supported the roles of miRNA in gene regulation upon chilling stress. Not only miRNAs but also tasiARFs were found to be differentially expressed in chillingstressed condition, indicating a perturbed small RNA system that is potentially associated with the widely altered transcriptome. As a caveat, additional experiments were required to confirm the results from the RNA-seq data.

\section{Identification of miRNAs and siRNAs in Cassava and castor bean}

In addition to an initial set of Cassava miRNAs we identified [30], a substantial number of miRNAs has been reported recently, based on deep-sequencing based profiling or computational prediction [31-33]. Nevertheless, we were able to identify 22 novel miRNAs in Cassava in the current study. Importantly, three novel miRNAs were highly expressed (Table 1A), indicating their important function in Cassava. Furthermore, we used qRT-PCR to validate 4 of the miRNAs that were computationally predicted in previous studies [31] (Additional file 2: Table S4B). We also examined the rest of the candidate miRNAs reported in [31]; however, only a few passed our miRNA identification criteria (see Methods). We thus expect the total number of miRNAs in Cassava to be close to what we report here, whereas the previous study has inflated the number of miRNAs in Cassava.

Here, we identified three TAS3 paralogs in both Cassava and castor bean genomes. While TAS3a is indicated in [13], TAS3b and TAS3c were newly identified in the two genomes in the current study. TAS3c encodes different tasi-ARF siRNAs from TAS3a and TAS3b, and is well conserved in Cassava and castor bean (Figure 2). A careful examination of ta-siRNAs and cis-NATs reported in the previous study [44] showed that the candidates satisfy neither of the two criteria: 1) the enrichment of 21-nt reads within a candidate locus; 2) unique mapping to the candidate locus.

\section{Differentially expressed sncRNA and auxin response under chilling stress}

A recurring theme from our sncRNA profiling and analysis was the set of auxin response genes. For example, miR167 and miR393 were reported to be dysregulated in Cassava of the current study and A. thaliana and wheat $[40,42,43]$. More importantly, miR167 has been reported to target auxin response factors, important transcription factors that regulate auxin response genes. Besides, dysregulated miR393 targets auxin signaling F-box, an important auxin receptor gene in the auxin signaling pathway. miR396 has been reported to target growth regulating factors (GRF), known transcription factors that regulate cell proliferation in Arabidopsis leaves [45]. Moreover, In $A$. thaliana, miR165 and miR166 have been implicated in regulating a set of targets involved in stress which have enriched functions of vascular development and growth $[40,41]$. The common theme of these chilling-responsive miRNAs is the regulation of auxin response and growth regulating genes, which are central players of many important aspects of plant growth and development as well as response to environmental stresses [46-49].

For ta-siRNAs, tasiARFs are well known to target auxin response factors in a wide variety of plant species. tasiARFs are derived from well-conserved TAS3 genes targeted by miR390 in plants. As expected, three TAS3 loci and the targeting miRNA, i.e., miR390, were found to be conserved in Cassava and castor bean. Not only did miRNAs target Auxin response factors, e.g., miR167 targeted ARF8, but secondary tasi-ARFs originating from the three TAS3 genes targeted auxin response factors as well, e.g., tasiARF1 and tasiARF3 targeted ARF3 (002399 m) (Figure 4). We showed that tasiARF1 also targeted auxin response factor $4(001979 \mathrm{~m})$. Overall, most validated target genes of siRNAs were related to receptors of Auxin signaling, transcription factors and down-stream receptor of Auxin related genes (Table 4). Besides, miR393-mediated auxin signaling F-box (AFB) genes in Arabidopsis and our current study on Cassava 
can further produce siRNAs, which potentially carry out a self regulation on AFB genes [16]. Taken together, both dysregulated miRNAs and siRNAs triggered by miRNAs regulate auxin-related genes, reflecting the robustness and plasticity of small RNA-mediated gene expression regulation.

Auxin is intimately involved in differential growth and bending. For example, root gravitropic curvature is driven by an asymmetric distribution of auxin response pathway [50]. Under a biotic stress, repressing of auxin signaling by miR393 has been shown to contribute to antibacterial resistance to P. syringae in Arabidopsis [51]. Under an abitoic stress, it has been shown that chilling stress affects the polar transport of auxin by selective inhibition of intracellular trafficking of auxin efflux carriers [50]. The abnormal auxin among cell, shoot or root and other developmental organs might result in the abnormal development during chilling stress, such as growth retardation, reproduction attenuation and a lack of common survival symptoms observed during chilling attack. Regulation of various factors by miRNAs in the auxin signaling pathway may indicate a conserved role of miRNAs in response to abiotic and biotic stress.

\section{Cold-responsive miRNAs regulate other important cellular} processes in response to chilling stress

In the companion publication [29], we identified a large number of anti-correlated pairs of differentially expressed miRNAs and DE mRNAs. The transcriptome analysis has shown that perturbations of small RNAs as well as variations of transcriptome were most prominent in the CS condition. miRNA-mediated targets were enriched in biosynthetic process, cellular protein modification process, response to stress and metabolic process, consistent with the results from another recent study of Cassava chilling-stressed transcriptome [52]. Particularly, multiple genes in the same enriched processes were regulated by miRNAs under multiple stress conditions. For example, 5 genes $(005409 \mathrm{~m}, 006360 \mathrm{~m}, 006048 \mathrm{~m}$, $005437 \mathrm{~m}$ and $005421 \mathrm{~m}$ ) targeted by miR399 function in the same biosynthetic process in CA vs. NC. One gene $(012052 \mathrm{~m})$ was involved in oxidation-reduction process and 3 genes $(033858 \mathrm{~m}, 014142 \mathrm{~m}$ and $000730 \mathrm{~m})$ in metabolic process under CCA vs. NC, which were potentially targeted by $\mathrm{miR} 396 \mathrm{a} / \mathrm{b} / \mathrm{c} / \mathrm{d}$ (Additional file 2). Surprisingly, one translation-related gene $(018488 \mathrm{~m})$ regulated by miR172 was differentially expressed in four comparisons: CA vs. NC, CS vs. NC, CCA vs. CA and CS vs. CCA. The regulatory roles of miR172 in CA and CS, but not in NC and CCA, may indicate that chillingacclimated Cassava may reduce energy expense during chilling acclimation and depend on reserved nutrients to combat the adverse effects of chilling stress. In short, miRNAs are indispensable regulating factors for low temperature adaptation in Cassava.

\section{Secondary siRNAs from disease-related genes}

The current study indicated that the majority of secondary siRNA-generating genomic loci correspond to conserved protein-coding genes in Cassava and castor bean. Recent studies have also revealed that a large number of secondary siRNAs, arranged in phasing as ta-siRNAs, arise from disease resistance NBS-LRR-encoding genes initiated by miRNA binding. In this scenario, several master miRNAs regulate a large number of genes in the NBS-LRR gene family. For example, miR1507, miR2118 and miR2109 in Medicago target more than 100 NBLRR-encoding genes to subsequently produce ample siRNAs $[18,19]$. Such a siRNA-yielding phenomenon has also been observed in A. thaliana and S. lycopersicum (tomato) [53-56]. Moreover, different plant species utilize different miRNAs to target genes in the same classes (e.g., NBS-LRR class) for siRNA production. For example, sly-miR482 and sly-miR2118 in tomato and athmiR472 and ath-miR825 in Arabidopsis all target a large number of NBS-LRR genes.

In addition, Cassava miR162 targeted Dicer-like 1 protein and initiated a small amount of siRNAs, which is consistent with the observation in Arabidopsis [57]. Remarkably, a large number of miRNA-medicated siRNAgenerating loci belong to the coding regions of genes of immune and stress response functions, particularly those disease resistant genes containing the NB-ARC domain. Taken together, a broadly conserved mechanism exists between miRNAs and their target genes in plants; moreover, secondary siRNAs that are derived from the target genes can accumulate to a prominent level, indicating their important function in plants

\section{Natural-antisense siRNAs}

Endo-siRNAs derived from cis-NATs have been widely reported in animal and plants. In Arabidopsis, nat-siRNAs from the SRO5 and P5CDH gene pair is induced by salt stress [20]. P5CDH is known as a stress-related gene while the function of SRO5 remains unknown [20]. Other natsiRNAs from ATGB2 and PPR only accumulates in response to a bacterial pathogen infection. In Arabidopsis and rice [22], genome-wide analysis of nat-siRNAs further suggested that the accumulation of many nat-siRNAs is condition-specific [25-27]. Therefore, chilling acclimation and chilling shock were anticipated to induce nat-siRNAs to negate the damage caused by the stress. In the current study, we identified several candidate cis-NAT-siRNA loci which yield a few number of small RNA reads only within the overlapping regions. One of the cis-nat-siRNAs was further found to be differentially expressed under chilling stress compared to the normal condition. 


\section{Conclusion}

Our study provided the first results on gene regulation by sncRNAs in chilling acclimation of Euphorbiaceous plants. We identified 83 (61 and 22) novel miRNAs as well as 78 (57 and 21) putative secondary siRNAyielding and 8 (7 and 1) nat-siRNA-yielding candidate loci in Cassava and castor bean, respectively. We showed above three sncRNA species and mRNA genes in Euphorbiaceous plants experienced dramatic change, especially Auxin response genes, after severe and moderate chilling stresses. This work laid a foundation to elucidate further function of those sncRNA-mediated pathways during chilling stress and acclimation in Cassava.

\section{Methods}

\section{Plant materials and stress treatments}

Stem segments with three nodes of Cassava (Manihot esculenta Crantz) cultivar, were cut from 8-month-old plants, and inclined in 3-L pots filled with barren red soil: vermiculite $(1: 1, \mathrm{v} / \mathrm{v})$, fertilized with Hoagland's solution [58], to propagate and generate well-balanced seedlings. The solution was renewed with $300 \mathrm{ml}$ quarterstrength solution once a week. After 2 months of planting, the uniform seedlings were subjected to chilling stress treatment. All plants were field grown at the Institute of Tropical Bioscience and Biotechnology (ITBB), Chinese Academy of Tropical Agricultural Sciences (CATAS), Haikou, during April and June of natural conditions (11 h light, $13 \mathrm{~h}$ dark and $25^{\circ} \mathrm{C}$ during the day and $18^{\circ} \mathrm{C}$ at night).

Two Cassava cultivars (SC124 and C4) were transferred to normal $24^{\circ} \mathrm{C}$ illumination incubator (SANYO, Japan) for 2 days to set a homogenization starting point, and then subjected to three types of chilling treatment. 1) Gradual chilling acclimation (CA in Figure 1): Temperature was decreased from $24^{\circ} \mathrm{C}$ to $14^{\circ} \mathrm{C}$ with the rate of $-2^{\circ} \mathrm{C} / \mathrm{h}$ to induce mild chilling stress. Temperature was then held constant at $14^{\circ} \mathrm{C}$ for five days to accommodate chilling acclimation. RNA was collected at $6 \mathrm{~h}, 24 \mathrm{~h}$ and $5 \mathrm{~d}$ after the temperature reaching $14^{\circ} \mathrm{C}$. 2) Cold stress after chilling acclimation (CCA): After 5 days of chilling acclimation and growth under $14^{\circ} \mathrm{C}$, plants were watered once with Hoagland's solution, transferred further to $4^{\circ} \mathrm{C}$ by $-2^{\circ} \mathrm{C} / \mathrm{h}$ gradient cooling, and cultivated at constant $4^{\circ} \mathrm{C}$ for another 5d. 3) Cold shock (CS): Plants grown under $24^{\circ} \mathrm{C}$ were subjected to dramatic temperature decline to $4^{\circ} \mathrm{C}$ with a rate of $-4^{\circ} \mathrm{C} / \mathrm{h}$ to make sure the temperature reached $4^{\circ} \mathrm{C}$ at the same time as the CCA treatment. In these two latter treatments, RNA was collected at $6 \mathrm{~h}$, $24 \mathrm{~h}$ and $5 \mathrm{~d}$ after temperature reaching $4^{\circ} \mathrm{C}$. In parallel, plants grown under the normal condition (NC) of $24^{\circ} \mathrm{C}$ were watered once with Hoagland's solution every 5 days, and RNA was collected at $0 \mathrm{~d}, 5 \mathrm{~d}$ and $10 \mathrm{~d}$. At the same time, the NC and CCA treatments of castor bean (cultivar
Hela) were carried out as for Cassava. The mixture samples of SC124 (details are given below) were subjected to small-RNA and mRNA expression profiling by NextGen deep sequencing.

\section{RNA isolation, RNA library preparation and NextGen deep sequencing}

Three organs/tissues (folded leaf, fully expanded leaf and roots) from three different Cassava cultivar SC124 plants harvested at $6 \mathrm{~h}, 24 \mathrm{~h}$ and $5 \mathrm{~d}$ for three chilling treatments of CA, CCA and CS, for profiling genes at the stages of initial response, secondary response, and functional adaption to chilling stresses. Total RNA of each sample was isolated individually, and then pooled with an equal amount from each sample into one for profiling. As a result, four mRNA libraries and four smallRNA libraries, corresponding to the conditions of CA, CCA, CS and NC, were constructed. Similarly, two castor bean small-RNA libraries for the CCA and NC conditions were prepared.

The six small-RNA libraries (four Cassava samples from the NC, CA, CCA and CS conditions plus two castor bean samples from the $\mathrm{NC}$ and CCA conditions) were subjected to small-RNA deep sequencing using Illumina GAII. Briefly, total RNA was isolated using RNAplant Reagent kit (TIANGEN, Beijing, China). Small RNAs were enriched by poly-ethylene glycol precipitation, separated on $15 \%$ denaturing PAGE, and visualized by SYBR-gold staining. Small RNAs of 16- to 28-nt were gel-purified. Purified small RNAs were ligated to a 5 'adaptor and a 3' adaptor sequentially, reverse transcription amplified, and sequenced.

The four mRNA libraries were sequenced by RNA-seq by Solexa GAII following Illumina RNA-seq protocol. Briefly, total RNAs were isolated, purified and reverse transcribed, the resulting cDNA products were subsequently digested with NlaIII and the $3{ }^{\prime}$-cDNA fragments captured with the oligo(dT) beads, and then ligated to the Illumina GEX NlaIII Adapter 1. The junction of Illumina adapter 1 and CATG site contained the recognition site of MmeI, which cut 17-nt downstream of the recognition site (CATG) to produce tags. After removing 3 ' fragments with magnetic beads precipitation and MmeI digestion, an Illumina GEX adapter 2 was introduced at the end of tags. The resulting adapter-ligated cDNA tags were subjected to 15 cycles of linear PCR amplified, purified and sequenced with the method of sequencing by synthesis (SBS) using the Illumina Genome Analyzer.

\section{Preprocessing of small RNA sequencing data}

Raw sequence reads that contained no 3' sequencing adaptor, were of low quality, or were shorter than 17-nt were discarded. The adaptor trimming was done by an inhouse method that recursively searches for the longest 
substring of the adaptor appearing within a sequence read. If a raw sequence read did not have a substring of the adaptor longer than 6-nt, it was considered to have no adaptor. The adaptor-trimmed sequences with no ambiguous reads, which were referred to as qualified reads, were then mapped to the Cassava genome using Bowtie [59].

\section{Identification of novel miRNAs}

Methods for novel miRNA identification were described in our previous papers on miRNAs in other plant species $[60,61]$. Here, we briefly discuss the key steps. First, qualified reads in all libraries that mapped to known Cassava (or castor bean) miRNAs (miRBase release 20) were excluded from the identification of novel miRNAs, but were used for re-annotation of known miRNAs. We then mapped the remaining reads to the Cassava genome (or castor bean genome) using Bowtie [59] and merged neighboring loci if they shared overlapping reads. The (merged) loci were extended 300-nt on both ends, and a series of segments of 250-nt were extracted in a sliding window fashion starting from the 5 '-end. We then examined the folding structures of the segments using RNAfold [62]. Candidate miRNAs were chosen based on four key criteria including presence of more than 10 reads, hairpin structures, appearance of miRNA* sequences and RNA-RNA duplex structures with $\sim 2$-nt $3^{\prime}$ overhangs.

\section{Identification of secondary siRNAs}

We searched for clusters with 21-nt reads enriched in genomic and cDNA sequences as secondary siRNAyielding candidates. Specifically, qualified reads from all small-RNA libraries that were aligned to miRNA loci were removed first. The remaining reads were then aligned to genomic and cDNA sequences with Bowtie (version 0.12.7) [59] allowing no mismatches. Genome-aligned reads were clustered within a window size of 50 base pairs to form a putative candidate region. Two stringent criteria were applied to those candidate transcripts and regions with mapped reads. First, candidates with less than 10 mapped reads were removed to ensure a sufficient level of expression. Second, the majority (over 70\%) of the mapped reads within a candidate transcript were ensured to be 21-nt long. The two criteria aimed to filter out false positive candidates due to random RNA degradation or other types of endogenous small RNAs that do not possess the characteristic of 21-nt enrichment. These criteria were previously adopted in rice and Arabidopsis [16,63].

We then used TargetFinder [64] to identify putative binding sites of a miRNA on mRNA transcripts. A pair of predicted binding sites on a transcript define a region that serves as a template for synthesizing a dsRNA for siRNA production [8-11]. Candidate siRNA-yielding transcripts were extracted with a flanking extension of 100-nt.
Every known and novel miRNA was subject to this analysis. We only considered binding sites with a score from TargetFinder of no less than 4 (see [64] for detail of the scoring metric).

\section{Identification of cis-NAT pairs and cis-nat-siRNAs}

We searched for anti-sense pairs of transcripts (aka, cis-NATs) in the Cassava genome [36] and the castor bean genome [65] which overlapped at least 25-nt at the same genomic loci. cis-NATs were further categorized into three groups: convergent ( $3^{\prime}-3^{\prime}$ overlap), divergent ( $5^{\prime}-5^{\prime}$ overlap), and enclosed where one transcript was entirely encompassed by the other [25]. We set three stringent criteria for identifying cis-NAT siRNAs, 1) the enrichment of 21-nt reads within a candidate locus; 2 ) presence of reads uniquely mapped to the candidate locus, and 3) more than 10 reads from the locus. The criteria removed false positive that may be degradation products.

\section{Identification of differentially expressed sncRNAs}

Reads that aligned perfectly to the candidate sncRNAyielding transcripts were used to compute the digital expression levels of the sncRNAs. Reads mapped to multiple genomic loci were attributed to all derivative small RNAs. Read counts in each sample were normalized to adjust for sample variations. Let $T$ be the number of qualified reads that aligned to the genome and cDNA sequences in that sample and $C$ the average value of $T$ of all samples. The normalized number of reads for each sncRNA in each sample is $\left(N_{s m R N A} * C / T\right)$, where $N_{\text {SmRNA }}$ is the number of raw sequencing reads of the sncRNA. Differentially expressed miRNAs were those that had at least 1.5 -fold change across two conditions compared.

\section{Identification of siRNA targets}

Every siRNA with more than 10 sequencing reads was subject to target prediction against cDNA sequences. The reverse complementary sequence of a siRNA was mapped to the cDNA sequences using Bowtie; those sites with no more than three mismatches were considered as putative targets of the siRNA.

\section{Identification of differentially expressed mRNA genes}

Sequencing reads from RNA-seq were aligned to the cDNA sequences of Cassava or castor bean using Bowtie (version 0.12.7) allowing no more than one mismatch. The number of mapped reads on each transcript was recorded as a raw read count. A gene was considered as expressed if it has at least 10 CPM (Count Per Million) mapped reads. The genes that had a CPM less than 10 were considered as not expressed. We then normalized the raw read counts of expressed genes using the upperquartile normalization method. Given two conditions to 
be compared, a gene was considered to be differentially expressed if either one of the following criteria was satisfied: (1) its normalized count changed at least 4 folds, or (2) the gene was not expressed under one condition but was expressed with more than $40 \mathrm{CPM}$ in the other condition.

\section{Identification of pairs of anti-correlated sncRNAs and targets}

A pair of a sncRNA (a miRNA, secondary siRNA or cis-nat-siRNA) and its target was considered as anticorrelated if the sncRNA was up- or down-regulated and the target was, respectively, down- or up-regulated in the two conditions compared. To filter out possible false positive anti-correlated sncRNA and target pairs we chose targets whose expression changed by at least 2 fold.

\section{Experimental validation and analysis of sncRNA}

The RNAplant Reagent kit (TIANGEN, Beijing, China) was used for total RNA isolation. The quantity and quality of extracted total RNAs was detected by $1 \%$ agarose gel electrophoresis and spectrophotometer. This same total RNA sample was used in small RNA and mRNA differential expression assays. A multiplexed RT method was applied to assess the differential expression of selected differential miRNAs. The total RNAs were firststrand cDNA synthesized with pool of miRNA-specific RT primers. These RT primers contain unique tag sequences at the $5^{\prime}$-ends and 7 - to 10 -nt complementary nucleotides with $3^{\prime}$ ends of specific miRNAs. Real-time PCR was then performed with the cDNA templates generated from the multiplexed $\mathrm{RT}$ reaction. The PCR reverse primer specifically anneals to the $5^{\prime}$-end of the CDNA templates, and the PCR forward primer specifically anneals to the tag sequence used in the RT primer. The forward and reverse primers were designed following the strategy by [66], which was developed to amplify mature miRNAs. The amplicons included 21- to 25-nt miRNA specific primers and 30-nt adaptors designed for the common reverse primer template, resulting in $\sim 55$-nt target length. The sequences of PCR primers are listed in Additional file 2: Table S13.

Three organs/tissues (folded leaf, fully expanded leaf and roots) from three different Cassava cultivar SC124 plants harvested at $6 \mathrm{~h}, 24 \mathrm{~h}$ and $5 \mathrm{~d}$ for control and three chilling treatments of CA, CCA and CS, as profiling samples. miRNAs and U6 as the reference gene for each sample were amplified in parallel for 3 replicates. The values of the threshold cycle (CT) were calculated using Rotor-Gene 6000 series software 1.7 (Corbett Robotics, Australia). CT values were converted to relative expression by the $\Delta \Delta \mathrm{CT}$ method with the following formula: The relative concentration was $2^{-\Delta \Delta C T}$, where $\Delta \Delta \mathrm{CT}=$ ( $\Delta$ CTsample $-\Delta$ CTcontrol), $\Delta \mathrm{CT}=\mathrm{CT}($ miRNA)-CT (U6) in each sample. If the $\mathrm{CT}$ value was greater than that of one with no template control (NTC), the miRNA was considered not expressed.

\section{Experimental miRNA target validation}

RNA ligase-mediated rapid amplification of 5'cDNA ends (RLM-RACE) GeneRacer Kit (Invitrogen, USA) was used to validate miRNA-guided mRNA cleavage, which differed with traditional 5'RACE of full-length cDNA by omitting the $5^{\prime}$ phosphates of truncated mRNA removal and the 5' cap structure of full-length mRNA removal treatments. Briefly, total RNA was extracted with RNAplant regent (TIANGEN, DP407-01), and PolyA RNA was isolated using polyAtract mRNA isolation system III (Promega, USA) to eliminate contaminated non-mRNA. Ligation with a 5' RNA adapter and a reverse transcription were performed then after. The resulting cDNA was used as a template for PCR amplification. Two 100 bp-spaced gene-specific reverse primers (GSP1 and GSP2) for each target were designed based on the downstream sequence of the miRNA target binding site at the target gene sequence, and combined with two GeneRacer 5' forward primers (included in GeneRacer kit) to specifically nest amplify the 3 ' cleavage product of the target mRNA. The amplified PCR products were gel purified, cloned and sequenced (Sangon, China). Gene specific primers that we used are provided in Additional file 2: Table S14.

\section{Supporting data}

The raw sequencing and processed data from this project have been deposited to the GEO database (http:// www.ncbi.nlm.nih.gov/geo/) under the accession number GSE52178.

\section{Additional files}

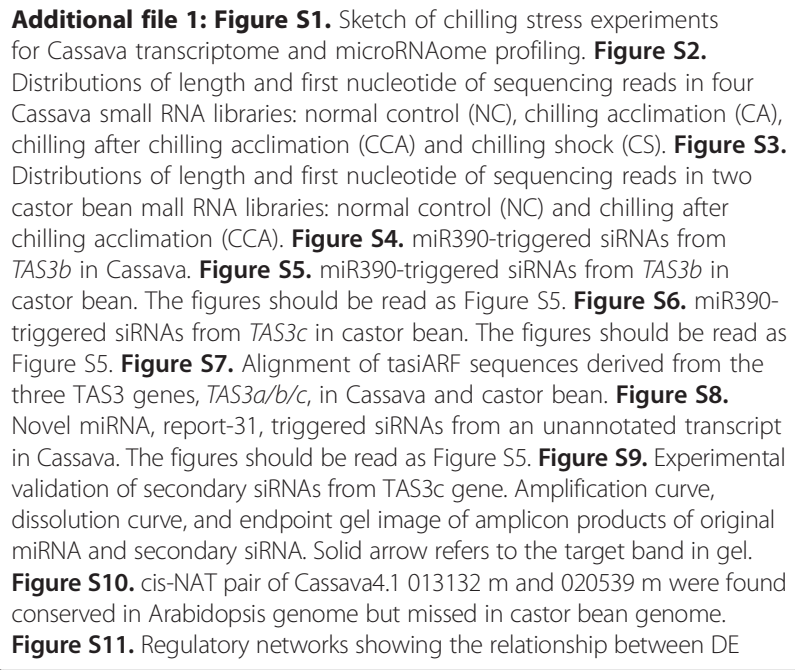


siRNAs and their anti-correlated target mRNAs. The diamonds indicate siRNA and the circles indicate target mRNAs.

Additional file 2: Table S1. Statistics of raw sequence reads from four small-RNA libraries from Cassava (A, B and C) under chilling stress and normal condition. Table S2. Statistics of RNA-seq data (raw reads and reads mapped to the reference genome with zero mismatches), expressed mRNAs and differentially expressed mRNAs from the normal condition (NC) and three chilling stress conditions (CA, CCA and CS). Table S3. Statistics of raw sequence reads from two small-RNA libraries from castor bean under chilling stress and normal condition. Table S4. A total of 93 individual miRNAs from 22 families in Cassava were identified by an extended analysis of previously detected miRNAs in Cassava. Table S5. Re-annotation of known miRNAs in castor bean. Table S6. The experimental validation of known, novel, and some miRNA partners under four chilling treatments in Cassava. Table S7. Secondary siRNAs derived from Cassava genes initiated by miRNA targeting. Table S8. Secondary siRNAs derived from castor bean genes initiated by miRNA targeting. Table S9. Cis-NAT pair loci information in Cassava genome. Table S10. Cis-NAT pair loci information in castor bean genome. Table S11. RNA-seq signals for the pair of cis-NAT genes in the four conditions. Table S12. Differentially expressed sncRNAs in Cassava identified in small RNA sequencing libraries. Table S13. The sequences of qRT-PCR primers used in this study. Table S14. The two gene specific primers (GSP1 and GSP2) used in the detection of cleavage site of miRNA on it corresponding target genes by 5'RACE experiment.

Additional file 3: Alignment of reads to novel Cassava miRNAs. Additional file 4: Alignment of reads to novel castor bean miRNAs. Additional file 5: Alignment of reads to known Cassava miRNAs. Additional file 6: Alignment of reads to known castor bean miRNAs.

\section{Competing interest}

The authors declare that they have no conflict of interest.

\section{Authors' contributions}

WZ and MP initiated the project and designed the experiments; $C Z$ carried out the experiments; JX and ZC performed the computational analyses; JX, WZ, ZC and CZ wrote the paper. Other authors from ITBB help experimental validation, and other authors from Jianghan University and WUSTL helped with computational analysis. All authors have read and approved the manuscript for publication.

\section{Acknowledgments}

The work was supported in part by Chinese National Basic Research and Development Program (973) (2010CB126602), Natural Science Foundation of China (31300999 and 31101193), National Nonprofit Institute Research Grant of CATAS-ITBB (ITBB140101), the municipal government of Wuhan, Hubei, China (2014070504020241 and the Talent Development Program), the Hainan Province Natural Science Foundation (313075), the Major Technology Project of Hainan (ZDZX2013023-1), and an internal research grant of Jianhan University, Wuhan, China, as well as by United States National Institutes of Health (R01GM100364) and United States National Science Foundation (DBI-0743797).

\section{Author details}

'Institute for Systems Biology, Jianghan University, Wuhan, Hubei 430056 , China. ${ }^{2}$ The Institute of Tropical Bioscience and Biotechnology, Chinese Academy of Tropical Agricultural Sciences, Haikou, China. ${ }^{3}$ Department of Computer Science and Engineering, Washington University in St. Louis, One Brookings Drive, St. Louis, MO 63130, USA. ${ }^{4}$ Department of Genetics, Washington University School of Medicine, St. Louis, MO 63110, USA.

\section{Received: 31 May 2014 Accepted: 15 July 2014}

Published: 29 July 2014

\section{Reference}

1. Baulcombe D: RNA silencing in plants. Nature 2004, 431:356-363.

2. Matzke MA, Birchler JA: RNAi-mediated pathways in the nucleus. Nat Rev Genet 2005, 6:24-35.
3. Chapman EJ, Carrington JC: Specialization and evolution of endogenous small RNA pathways. Nat Rev Genet 2007, 8:884-896.

4. Jones-Rhoades MW, Bartel DP, Bartel B: MicroRNAs and their regulatory roles in plants. Annu Rev Plant Biol 2006, 57:19-53.

5. Zamore PD, Haley B: Ribo-gnome: the big world of small RNAs. Science 2005, 309:1519-1524.

6. Carthew RW, Sontheimer EJ: Origins and Mechanisms of miRNAs and siRNAs. Cell 2009, 136:642-655.

7. Allen $E$, Howell MD: miRNAs in the biogenesis of trans-acting siRNAs in higher plants. Semin Cell Dev Biol 2010, 21:798-804.

8. Mosher RA, Melnyk CW, Kelly KA, Dunn RM, Studholme DJ, Baulcombe DC: Uniparental expression of PolIV-dependent siRNAs in developing endosperm of Arabidopsis. Nature 2009, 460:283-286.

9. Wierzbicki AT, Haag JR, Pikaard CS: Noncoding Transcription by RNA Polymerase Pol IVb/Pol V Mediates Transcriptional Silencing of Overlapping and Adjacent Genes. Cell 2008, 135(4):635-648.

10. Pikaard CS, Haag JR, Ream T, Wierzbicki AT: Roles of RNA polymerase IV in gene silencing. Trends Plant Sci 2008, 13(7):390-397.

11. Rajagopalan R, Vaucheret H, Trejo J, Bartel DP: A diverse and evolutionarily fluid set of microRNAs in Arabidopsis thaliana. Genes Dev 2006, 20:3407-3425.

12. Vazquez F: Arabidopsis endogenous small RNAs: highways and byways. Trends Plant Sci 2006, 11(9):460-468.

13. Axtell MJ, Jan C, Rajagopalan R, Bartel DP: A two-hit trigger for siRNA biogenesis in plants. Cell 2006, 127(3):565-577.

14. Ronemus M, Vaughn MW, Martienssen RA: MicroRNA-Targeted and Small Interfering RNA-Mediated mRNA Degradation Is Regulated by Argonaute, Dicer, and RNA-Dependent RNA Polymerase in Arabidopsis. Plant Cell Online 2006, 18(7):1559-1574.

15. Cuperus JT, Fahlgren N, Carrington JC: Evolution and functional diversification of MIRNA genes. Plant Cell 2011, 23(2):431-442.

16. Howell MD, Fahlgren N, Chapman EJ, Cumbie JS, Sullivan CM, Givan SA, Kasschau KD, Carrington JC: Genome-Wide Analysis of the RNADEPENDENT RNA POLYMERASE6 / DICER-LIKE4 Pathway in Arabidopsis Reveals Dependency on miRNA- and tasiRNA-Directed Targeting. Plant Cell Online 2007, 19(3):926-942.

17. Lu C, Jeong D-H, Kulkarni K, Pillay M, Nobuta K, German R, Thatcher SR, Maher C, Zhang L, Ware D, Liu B, Cao X, Meyers BC, Green PJ: Genomewide analysis for discovery of rice microRNAs reveals natural antisense microRNAs (nat-miRNAs). Proc Natl Acad Sci 2008, 105(12):4951-4956.

18. Shivaprasad PV, Chen H-M, Patel K, Bond DM, Santos B a CM, Baulcombe DC: A microRNA superfamily regulates nucleotide binding site-leucinerich repeats and other mRNAs. Plant Cell 2012, 24:859-874.

19. Zhai J, Jeong D-H, De Paoli E, Park S, Rosen BD, Li Y, González AJ, Yan Z, Kitto SL, Grusak M a, Jackson S a, Stacey G, Cook DR, Green PJ, Sherrier DJ, Meyers BC: MicroRNAs as master regulators of the plant NB-LRR defense gene family via the production of phased, trans-acting siRNAs. Genes Dev 2011, 25:2540-2553.

20. Borsani O, Zhu J, Verslues PE, Sunkar R, Zhu J-K: Endogenous siRNAs Derived from a Pair of Natural cis-Antisense Transcripts Regulate Salt Tolerance in Arabidopsis. Cell 2005, 123(7):1279-1291.

21. Jin H: Endogenous small RNAs and antibacterial immunity in plants. FEBS Lett 2008, 582:2679-2684.

22. Katiyar-Agarwal S, Morgan R, Dahlbeck D, Borsani O, Villegas A, Zhu J-K, Staskawicz BJ, Jin $\mathrm{H}$ : A pathogen-inducible endogenous siRNA in plant immunity. Proc Natl Acad Sci 2006, 103:18002-18007.

23. Ron M, Alandete Saez M, Eshed Williams L, Fletcher JC, McCormick S: Proper regulation of a sperm-specific cis-nat-siRNA is essential for double fertilization in Arabidopsis. Genes Dev 2010, 24:1010-1021.

24. Zubko E, Meyer P: A natural antisense transcript of the Petunia hybrida Sho gene suggests a role for an antisense mechanism in cytokinin regulation. Plant J 2007, 52:1131-1139.

25. Zhou X, Sunkar R, Jin H, Zhu J-K, Zhang W: Genome-wide identification and analysis of small RNAs originated from natural antisense transcripts in Oryza sativa. Genome Res 2009, 19(1):70-78.

26. Zhang X, Xia J, Lii Y, Barrera-Figueroa B, Zhou X, Gao S, Lu L, Niu D, Chen Z, Leung C, Wong T, Zhang H, Guo J, Li Y, Liu R, Liang W, Zhu J-K, Zhang W, Jin $\mathrm{H}$ : Genome-wide analysis of plant nat-siRNAs reveals insights into their distribution, biogenesis and function. Genome Biol 2012, 13:R20.

27. Jin $\mathrm{H}$, Vacic $\mathrm{V}$, Girke $\mathrm{T}$, Lonardi S, Zhu J-K: Small RNAs and the regulation of cis-natural antisense transcripts in Arabidopsis. BMC Mol Biol 2008, 9:6. 
28. Katiyar-Agarwal S, Gao S, Vivian-Smith A, Jin H: A novel class of bacteriainduced small RNAs in Arabidopsis. Genes Dev 2007, 21:3123-3134.

29. Zeng C, Chen Z, Xia J, Zhang K, Chen X, Zhou Y, Bo W, Song S, Yang R, Yang Z, Guo X, Wang B, Peng H, Wang W, Peng M, Zhang W: Chilling acclimation provides immunity to stress by altering regulatory networks and inducing genes with protective functions in Cassava. BMC Plant Biology 2014. in press.

30. Zeng C, Wang W, Zheng Y, Chen X, Bo W, Song S, Zhang W, Peng M: Conservation and divergence of microRNAs and their functions in Euphorbiaceous plants. Nucleic Acids Res 2010, 38(3):981-995.

31. Ballén-Taborda C, Plata G, Ayling S, Rodríguez-Zapata F, Becerra LopezLavalle LA, Duitama J, Tohme J: Identification of Cassava MicroRNAs under Abiotic Stress. Int J Genomics 2013, 2013:857986.

32. Patanun $\mathrm{O}$, Lertpanyasampatha M, Sojikul P, Viboonjun U, Narangajavana J: Computational Identification of MicroRNAs and Their Targets in Cassava (Manihot esculenta Crantz.). Mol Biotechnol 2013, 53:257-269.

33. Perez-Quintero A, Quintero A, Urrego O, Vanegas P, Lopez C: Bioinformatic identification of Cassava miRNAs differentially expressed in response to infection by Xanthomonas axonopodis pv. manihotis. BMC Plant Biol 2012, 12:29

34. Van Ooijen G, Mayr G, Kasiem MMA, Albrecht M, Cornelissen BJC, Takken FLW: Structure-function analysis of the NB-ARC domain of plant disease resistance proteins. J Exp Bot 2008, 59(6):1383-1397.

35. Allen E, Xie Z, Gustafson AM, Carrington JC: microRNA-Directed Phasing during Trans-Acting siRNA Biogenesis in Plants. Cell 2005, 121(2):207-221.

36. Prochnik S, Marri P, Desany B, Rabinowicz P, Kodira C, Mohiuddin M, Rodriquez F, Fauquet C, Tohme J, Harkins T, Rokhsar D, Rounsley S: The Cassava Genome: Current Progress, Future Directions. Trop Plant Biol 2012, 5:88-94. LA - English.

37. Jain M, Nijhawan A, Arora R, Agarwal P, Ray S, Sharma P, Kapoor S, Tyagi AK, Khurana JP: F-Box Proteins in Rice. Genome-Wide Analysis, Classification, Temporal and Spatial Gene Expression during Panicle and Seed Development, and Regulation by Light and Abiotic Stress. Plant Physiol 2007, 143(4):1467-1483.

38. Lang P, Zhang C-K, Ebel RC, Dane F, Dozier W a: Identification of chilling acclimated genes in leaves of Citrus unshiu by mRNA differential display. Gene 2005, 359:111-118.

39. Hu R, Qi G, Kong Y, Kong D, Gao Q, Zhou G: Comprehensive Analysis of NAC Domain Transcription Factor Gene Family in Populus trichocarpa. BMC Plant Biol 2010, 10:145

40. Liu H-H, Tian X, Li Y-J, Wu C-A, Zheng C-C: Microarray-based analysis of stress-regulated microRNAs in Arabidopsis thaliana. RNA 2008, 14(5):836-843.

41. Zhou X, Wang G, Sutoh K, Zhu J-K, Zhang W: Identification of chillinginducible microRNAs in plants by transcriptome analysis. Biochim Biophys Acta - Gene Regul Mech 2008, 1779:780-788.

42. Tang Z, Zhang L, Xu C, Yuan S, Zhang F, Zheng Y, Zhao C: Uncovering Small RNA-Mediated Responses to Cold Stress in a Wheat Thermosensitive Genic Male-Sterile Line by Deep Sequencing. Plant Physiol 2012 159(2):721-738.

43. Lv D-K, Bai X, Li Y, Ding X-D, Ge Y, Cai H, Ji W, Wu N, Zhu Y-M: Profiling of chilling-stress-responsive miRNAs in rice by microarrays. Gene 2010, 459:39-47.

44. Quintero A, Pérez-Quintero AL, López C: Identification of ta-siRNAs and Cis-nat-siRNAs in Cassava and Their Roles in Response to Cassava Bacterial Blight. Genomics Proteomics Bioinformatics 2013, 11:172-181.

45. Debernardi JM, Rodriguez RE, Mecchia MA, Palatnik JF: Functional Specialization of the Plant miR396 Regulatory Network through Distinct MicroRNA-Target Interactions. PLoS Genet 2012, 8:e1002419.

46. Sakata T, Oshino T, Miura S, Tomabechi M, Tsunaga Y, Higashitani N, Miyazawa Y, Takahashi H, Watanabe M, Higashitani A: Auxins reverse plant male sterility caused by high temperatures. Proc Natl Acad Sci 2010, 107(19):8569-8574.

47. Sundberg E, Østergaard L: Distinct and Dynamic Auxin Activities During Reproductive Development. Cold Spring Harb Perspect Biol 2009, 1(6):a001628.

48. Vanneste S, Friml J: Auxin: A Trigger for Change in Plant Development. Cell 2009, 136(6):1005-1016.

49. Cecchetti V, Altamura MM, Falasca G, Costantino P, Cardarelli M: Auxin Regulates Arabidopsis Anther Dehiscence, Pollen Maturation, and Filament Elongation. Plant Cell Online 2008, 20:1760-1774.
50. Shibasaki K, Uemura M, Tsurumi S, Rahman A: Auxin Response in Arabidopsis under Cold Stress: Underlying Molecular Mechanisms. Plant Cell Online 2009, 21:3823-3838.

51. Navarro L, Dunoyer P, Jay F, Arnold B, Dharmasiri N, Estelle M, Voinnet O, Jones JD: A plant miRNA contributes to antibacterial resistance by repressing auxin signaling. Science 2006, 312:436-439.

52. An D, Yang J, Zhang P: Transcriptome profiling of low temperaturetreated Cassava apical shoots showed dynamic responses of tropical plant to chilling stress. BMC Genomics 2012, 13:64.

53. Manavella $P$, Koenig D, Weigel D: Plant secondary siRNA production determined by microRNA-duplex structure. Proc Natl Acad Sci U S A 2012, 109:2461-2466.

54. Lu C, Kulkarni K, Souret FF, MuthuValliappan R, Tej SS, Poethig RS, Henderson IR, Jacobsen SE, Wang W, Green PJ, Meyers BC: MicroRNAs and other small RNAs enriched in the Arabidopsis RNA-dependent RNA polymerase-2 mutant. Genome Res 2006, 16:1276-1288.

55. Cuperus JT, Carbonell A, Fahlgren N, Garcia-Ruiz H, Burke RT, Takeda A Sullivan CM, Gilbert SD, Montgomery TA, Carrington JC: Unique functionality of 22-nt miRNAs in triggering RDR6-dependent siRNA biogenesis from target transcripts in Arabidopsis. Nat Struct Mol Biol 2010, 17:997-1003.

56. Shivaprasad PV, Rajeswaran R, Blevins T, Schoelz J, Meins F, Hohn T, Pooggin MM: The CaMV transactivator/viroplasmin interferes with RDR6 dependent trans-acting and secondary siRNA pathways in Arabidopsis. Nucleic Acids Res 2008, 36:5896-5909.

57. Xie Z, Kasschau KD, Carrington JC: Negative Feedback Regulation of Dicer-Like1 in Arabidopsis by microRNA-Guided mRNA Degradation. Curr Biol 2003, 13:784-789.

58. Hoagland DR, Arnon DI: The water-culture method for growing plants without soil. Calif Dep Agric Exp Stn 1950. No. 347 (Berkeley, CA: University of California), pp. 1-32

59. Langmead B, Trapnell C, Pop M, Salzberg SL: Ultrafast and memoryefficient alignment of short DNA sequences to the human genome. Genome Biol 2009, 10:R25.

60. Sunkar R, Zhou XF, Zheng Y, Zhang WX, Zhu JK: Identification of novel and candidate miRNAs in rice by high throughput sequencing. BMC Plant Biol 2008, 8:25.

61. Zhang W, Gao S, Zhou X, Xia J, Chellappan P, Zhou X, Zhang X, Jin H: Multiple distinct small RNAs originate from the same microRNA precursors. Genome Biol 2010, 11:R81.

62. Hofacker IL: Vienna RNA secondary structure server. Nucleic Acids Res 2003, 31:3429-3431.

63. Johnson C, Kasprzewska A, Tennessen K, Fernandes J, Nan G-L, Walbot V, Sundaresan V, Vance V, Bowman LH: Clusters and superclusters of phased small RNAs in the developing inflorescence of rice. Genome Res 2009, 19(8):1429-1440.

64. Fahlgren N, Howell MD, Kasschau KD, Chapman EJ, Sullivan CM, Cumbie JS, Givan SA, Law TF, Grant SR, Dangl JL, Carrington JC: High-Throughput Sequencing of Arabidopsis microRNAs: Evidence for Frequent Birth and Death of MIRNA Genes. PLoS One 2007, 2:e219.

65. Chan AP, Crabtree J, Zhao Q, Lorenzi H, Orvis J, Puiu D, Melake-Berhan A, Jones KM, Redman J, Chen G, Cahoon EB, Gedil M, Stanke M, Haas BJ, Wortman JR, Fraser-Liggett CM, Ravel J, Rabinowicz PD: Draft genome sequence of the oilseed species Ricinus communis. Nat Biotech 2010, 28:951-956

66. Wang X: A PCR-based platform for microRNA expression profiling studies. RNA 2009, 15(4):716-723.

\section{doi:10.1186/1471-2164-15-634}

Cite this article as: Xia et al:: Endogenous small-noncoding RNAs and their roles in chilling response and stress acclimation in Cassava. BMC Genomics 2014 15:634 